\title{
Dominant Awn Inhibitor, which encodes an ALOG protein in sorghum, suppresses awn formation in rice
}

\author{
Hideki Takanashi ${ }^{1}$, Hiromi Kajiya-Kanegae ${ }^{1, \dagger}$, Asuka Nishimura ${ }^{1}$, Junko Yamada $^{1}$, Motoyuki Ishimori ${ }^{1}$, \\ Masaaki Kobayashi ${ }^{2}$, Kentaro Yano ${ }^{2}$, Hiroyoshi Iwata ${ }^{1}$, Nobuhiro Tsutsumi ${ }^{1, *}$, Wataru Sakamoto ${ }^{3, *}$
}

${ }^{1}$ Graduate School of Agricultural and Life Sciences, The University of Tokyo, 1-1-1 Yayoi, Bunkyo-ku, Tokyo 113-8657, Japan

${ }^{2}$ Department of Life Sciences, Faculty of Agriculture, Meiji University, Kawasaki, Kanagawa 214-8571, Japan

${ }^{3}$ Institute of Plant Science and Resources, Okayama University, Kurashiki, Okayama 710-0046, Japan

*Corresponding authors: saka@ okayama-u.ac.jp (W.S.) and atsutsu@g.ecc.u-tokyo.ac.jp (N.T.)

\section{Footnote}

${ }^{\dagger}$ Present address: Research Center for Agricultural Information Technology, National Agriculture and Food Research Organization, Minato-ku, Tokyo 105-0003, Japan

Running head: Dominant Awn Inhibitor in sorghum

Number of black and white figures: 0

Numbers of color figures: 6

Number of tables: 1

Number of supplementary figures: 3

Numbers of supplementary tables: 2 


\begin{abstract}
The awn, a needle-like structure that extends from the tip of the lemma in grass species, is believed to play a role in environmental adaptation and fitness. Wild species of cereal crops tend to have longer awns, whereas modern cultivars display a variety of awn lengths and morphologies. Awns in some crops seem to have been eliminated during domestication, and genes controlling awn formation have been reported. Interestingly, a few single dominant factors that eliminate awns are already known; in sorghum (Sorghum bicolor), for example, the dominant awn inhibitor has been known for a century, though its molecular identity remains uncharacterized. In this study, we conducted quantitative trait locus (QTL) analysis and a genome-wide association study of awn-related traits in sorghum. Among the three QTLs controlling awn presence and length, one of them, mapped on chromosome 3, had an extraordinary effect on eliminating awns. We provide a line of evidence demonstrating that this QTL corresponds to Dominant Awn Inhibitor (DAI), encoding an ALOG family protein that potentially acts as a transcription factor. Detailed analysis of the ALOG protein family in cereals revealed that $D A I$ was considered to originate from a gene duplication of its twin paralogue on chromosome 10 . Interestingly, heterologous expression of $D A I$ with its own promoter in rice inhibited awn formation in the awned cultivar Kasalath. Our finding that $D A I$ was born by gene duplication provides an interesting example of gain-of-function, which occurs only in sorghum but shares its functionality with rice and sorghum.
\end{abstract}

\title{
Keywords
}

Awn, Dominant Awn Inhibitor, genome-wide association study (GWAS), quantitative trait locus (QTL), sorghum 


\section{Introduction}

The awn is a needle-like structure that forms at the tip of the lemma in grass species. Awns, by their bristle-like architecture, are known to have various important functions, such as avoiding getting fed on by animals (Grundbacher 1963, Hua et al. 2015), helping seeds to burrow into the soil (Elbaum et al. 2007), and promoting seed dispersal (Sato et al. 1996, Cavanagh et al. 2020). Moreover, awns significantly contribute to photosynthesis and thereby to grain yield, especially in crops such as barley (Kjack and Witters 1974) and wheat (Motzo and Giunta 2002, Li et al. 2006). Wild species of cereal crops tend to have long awns, and numerous genetic studies in such crops have demonstrated that multiple factors regulate awn formation and elongation. Although important in wild species, awns in cereal crops often disturb modern agriculture by hindering manual harvesting and reducing the feed value of straw for livestock (Takahashi 1955). Consequently, awns have been partially or completely eliminated during domestication (Takahashi 1955).

Awn formation and elongation have been extensively characterized in rice at the molecular level, which indicates that the awnless cultivars were established by selecting the loss-of-function allele of these genes one after another during domestication (Amarasinghe et al. 2020, Bessho-Uehara et al. 2021). An-1/REGULATOR OF AWN ELONGATION 1 (RAE1) encodes the bHLH transcription factor (Luo et al. 2013, Furuta et al. 2015) expressed at the apex of lemma primordia, which causes continuous cell division for awn elongation. $L O N G$ and BARBED AWN1 (LABA1)/An-2 encodes a cytokinin-activating enzyme that positively regulates awn elongation ( $\mathrm{Gu}$ et al. 2015, Hua et al. 2015). RAE2/GAD1/GLA, which encodes epidermal patterning factor-like protein 1 (EPFL1), which acts as a small secretory peptide in panicles (Bessho-Uehara et al. 2016, Jin et al. 2016, Yano et al. 2016, Zhang et al. 2019). Two other genes, DROOPING LEAF (DL), encoding a YABBY gene and OSETTIN2 (OSETT2), encoding an auxin-responsive factor, also enhance awn elongation in rice (Toriba and Hirano 2014), although whether these genes are involved in domestication is unclear. In barley, short awn 2 (lks2, for length 2), which encodes a SHORT INTERNODES family transcription factor, has been shown to regulate awn length and pistil morphology (Taketa et al. 2011, Yuo et al. 2012). Genes involved in brassinosteroid biosynthesis or signaling have also been shown to affect awn length with pleiotropic effects (Dockter et al. 2014).

In contrast to these 'positive' factors for awn development, 'inhibitory' factors that diminish awn are also known, including Hooded (Hd), Tipped1 (B1), and Tipped2 (B2) in wheat (Watkins and Ellerton 1940, Kato et al. 1998, Sourdille et al. 2002, Yoshioka et al. 2017). Bl was recently found to encode a C2H2 zinc-finger protein with ethylene-responsive element binding factor-associated amphiphilic repression (EAR) motifs which putatively functions as a transcriptional repressor (DeWitt et al. 2020, Huang et al. 2020). Interestingly, such inhibitory factors have not been reported in rice, despite extensive studies. These results imply that awn formation is controlled by a complex and distinct genetic network, whose modification leads to varietal diversification in a species-specific manner.

Among cereal crop species, sorghum (Sorghum bicolor) is not an exception and displays a variety of awn morphologies among cultivars; however, unlike the aforementioned advanced studies, the genes responsible for awn development are poorly understood. Sorghum is the fifth most important cereal in the world (faostat.fao.org) and is a promising $\mathrm{C}_{4}$ crop with higher stress tolerance than other major cereals (Tuinstra et al. 
1997, Ogbaga et al. 2014). In addition, it is a suitable genetic model for $\mathrm{C}_{4}$ grasses because of its high morphological diversity and relatively small genome (approximately $732 \mathrm{Mb}$ ) compared to that in other $\mathrm{C}_{4}$ grasses (Price et al. 2005, Paterson et al. 2009, McCormick et al. 2018). In fact, our recent work showed that a next-generation sequencing approach, restriction site-associated DNA sequencing (RAD-seq), combined with the use of recombinant inbred lines (RILs), allowed the establishment of a high-density genetic map to elucidate important traits and their responsible genes, including genes associated with biomass, spikelet formation, seed morphologies, and response to chemicals (Kajiya-Kanegae et al. 2020, Jing et al. 2021, Takanashi et al. 2021). We reasoned that awn development could also be investigated, since the parents used in the RILs displayed contrasting awn morphologies. As for awn-related traits in sorghum, three quantitative trait locus (QTL) analyses (Boivin et al. 1999, Tao et al. 2000, Hart et al. 2001) and three genome-wide association studies (GWAS) (Shehzad et al. 2009, Zhang et al. 2015, Girma et al. 2019) have been previously reported, but their molecular identity remains unclear. It is noteworthy that the existence of a dominant awn inhibitor locus was reported a century ago (Vinall and Cron 1921, Sieglinger et al. 1934), suggesting the emergence of awnless cultivars in sorghum breeding.

In the present study, we conducted QTL analysis using RILs and GWAS using the sorghum germplasm for awn-related traits to enrich our limited knowledge on awn regulation in sorghum. Based on our QTL analysis for awn presence and awn length, we provide evidence that a single gene, Dominant Awn Inhibitor (DAI), located on chromosome 3, inhibits awn formation. DAI is likely to have originated from the duplication of its paralogue in chromosome 10, whose role remains unclear. Surprisingly, heterologous expression of sorghum $D A I$ into rice was shown to make the awned rice cultivar 'Kasalath' awnless. Together, these results suggest that common mechanisms may exist to inhibit awn formation in cereal crops, whereas the emergence of such inhibitors differs among species. 


\section{Results}

\section{Awned and awnless: morphology used in this study}

Sorghum has two types of spikelets: sessile spikelets (SSs) directly attached to the inflorescence branch, and pedicellate spikelets (PSs) attached to the inflorescence branch via a pedicel. Unlike PS, which has only incomplete floral organs, SS has a complete floret consisting of a lemma, palea, two lodicules, three anthers, and a pistil, therefore only SS can produce a seed. The awn, which is a slender extension of the lemma, is also born only in SS. Recently, we established and reported a sorghum RIL, derived from a parental cross between BTx623 and the Japanese landrace NOG (Kajiya-Kanegae et al. 2020). Since parents of this population differed in various traits (Ohnishi et al. 2019, Kajiya-Kanegae et al. 2020), we considered this RIL population to be suitable for various QTL analyses. Interestingly, we found that there was a large difference in awn length (AL) between the parents of our RILs (Fig. 1A and 1 B; BTx623 is awnless, while NOG is awned). Therefore, QTL analysis of the awn-related traits using this RILs was performed, using two generations $\left(\mathrm{F}_{6}\right.$ and $\left.F_{7}\right)$ cultivated in Okayama $\left(F_{6}\right.$, greenhouse, in 2014) and Tokyo ( $F_{7}$, field, in 2015) to validate the reproducibility.

\section{QTL analysis of the awn-related traits identified a prominent dominant allele that inhibits awn formation}

Measurement of the AL of 245 RIL individuals ( $\mathrm{F}_{6}$ generation) revealed that about half of the RILs are awnless like BTx623 (Fig. 1C). We then performed composite interval mapping of QTL using this AL as phenotypic data, with 1,789 SNP markers determined by RAD-seq (Kajiya-Kanegae et al. 2020). We detected an extraordinary high QTL on chromosome 3, termed $q A L 3$ [the logarithm of the odds (LOD) score 116.4 and the percentage of phenotypic variance explained (PVE) 90.6\%]. An additional QTL peak was found on chromosome 9 ( $q A L 9$ : LOD score 7.0, PVE 1.3\%) (Fig. 1D, Table 1). For $q A L 3$, the effect of increasing AL was attributed to the NOG allele (Fig. 1E, left). It is noteworthy that ALs are zero in almost all individuals carrying the BTx623 homozygous or heterozygous allele at $q A L 3$, strongly indicating its dominant effect on inhibiting awn formation or elongation. Confirming this, spikelets of $F_{1}$ plants derived from the cross between BTx623 and NOG were shown to have no awns (Supplementary Fig. 1A). For $q A L 9$, the effect for AL was not clearly resolved, most likely due to the superior effect of $q A L 3$ (Fig. 1E middle). Nevertheless, the NOG allele for $q A L 9$ appeared to increase AL, when the allele of $q A L 3$ was limited to only the NOG type (Fig. 1E right).

The results of our QTL analysis suggest that $q A L 3$ determines the awn presence (AP) rather than AL; therefore, we conducted QTL analysis using data converted from AL data to AP (re-scored as awnless = 0 , awned $=1$ ) to verify this. Again, we detected a single QTL on chromosome 3 (qAP3: LOD score 179.1, PVE 97.8\%), which had exactly the same position as $q A L 3$ but with a more intense effect (Fig. 1F, Table 1). The allelic effect of $q A P 3$ was similar as that of $q A L 3$; the BTx623 allele dominantly led to an awnless phenotype (Fig. 1G). Together, these results led us to conclude that the single locus corresponding to $q A L 3 / q A P 3$ is a dominant inhibitor of awn formation.

\section{Existence of two QTLs controlling awn length}


To enrich our knowledge on the control of AL, we performed QTL analysis using AL only in awned lines. By subtracting awnless individuals, we sought to exclude the extraordinary effect of $q A L 3 / q A P 3$ and to find other QTLs that may control AL. As expected, we were able to detect two additional QTLs on chromosome 7 (qAL7: LOD score 8.3, PVE 23.8\%) and 9 (qAL9: LOD score 5.9, PVE 16.2\%) (Fig. 1H, Table 1). Given that the property of $q A L 9$ detected here is quite similar to that of $q A L 9$ in the original QTL analysis with awnless individuals (Table 1), both were considered to represent the same QTL. Effects of increasing AL were attributed to the NOG alleles for both $q A L 7$ and $q A L 9$ (Fig. 1I). The QTLs presented here ( $q A L 3 / q A P 3, q A L 7$, and $q A L 9$ ) were also detected in the QTL analysis using the datasets of the 2015 cultivation in Tokyo, indicating high reproducibility for all QTLs (Supplementary Fig. 1B-H, Table 1). Based on these findings, the dominant inhibitory effect of $q A L 3 / A P 3$ is apparent, and we further characterized $q A L 3 / A P 3$ hereafter. As for $q A L 7$ and $q A L 9$, their presumed actions are described in Discussion.

\section{Reconfirming the effect of qAL3/qAP3 by establishing NIL}

Because several RILs were shown to be heterozygous for $q A L 3 / A P 3$ by RAD-seq in the $\mathrm{F}_{6}$ population, we next attempted to establish a near isogenic line (NIL). Of these, we selected an awnless heterozygous line at the $\mathrm{F}_{6}$ generation, RIL205 (Fig. 2A), and followed the segregation of $q A L 3 / A P 3$ in the subsequent selfed generations. Consequently, observations of spikelets of the NILs ( $F_{9}$ generation) showed that the NIL with the homogeneous BTx623 allele (NIL_B) lost awns, while that with the homogeneous NOG allele (NIL_N) had awns (Fig. 2B-E). These results suggest that there is indeed a gene in the qAL3/qAP3 locus that inhibits AL or AP. Dissection of immature spikelets in NIL_N indicated that awns appear before and after the length of the sessile spikelet reaches approximately $2.0 \mathrm{~mm}$ (between Stage 5 and 6; Fig. 2B), implying that the gene responsible for $q A L 3 / q A P 3$ may function at least around stage 5 .

\section{GWAS in the sorghum germplasm highlights the widespread distribution of qAL3/qAP3}

To clarify whether the extraordinary effectiveness of $q A L 3 / q A P 3$ is the only local QTL limited to our RIL population or is globally involved in awn regulation in sorghum, we conducted GWAS for AL using 289 accessions of sorghum germplasm, whose data were collected from cultivation in Tokyo for three years (2015, 2019, and 2020). Our germplasm collection was shown to exhibit a large variability in AL (Fig. 3A), and more than half of the accessions were awnless (Fig. 3B). First, we conducted GWAS using this AL data and found significant associations on chromosome 3 with a peak at Chr03:72641259 (Fig. 3C). This region was found to be consistent with that of the marker interval of $q A L 3 / q A P 3$, and the peak of GWAS and the nearest marker of qAL3/qAP3 were only $14 \mathrm{~kb}$ apart (Fig. 3D). The effect of the peak genotype was the same as that of qAL3/qAP3; ALs were zero in lines carrying the homozygous reference-type (BTx623) allele at the peak. In addition, similar results were observed for lines carrying the heterozygous allele at the peak (Fig. 3E). Next, we performed GWAS using the AP phenotype as we did in the QTL analysis, and detected similar associations on chromosome 3 as those detected in the AL (Fig. 3C insert). These results suggest that qAL3/qAP3 in the QTL analysis and the peak of GWAS are likely to represent the same gene. Detected associations of GWAS presented here were also found in GWAS using datasets of the 2019 and 2020 cultivation, and these results indicate high 
reproducibility for this association (Supplementary Fig. 1I and 1J). The fact that two reports have detected similar associations for AP on chromosome 3 (Zhang et al. 2015, Girma et al. 2019) by using another germplasm population also guarantees the validity of our results.

To assess the geographic distribution of alleles at the peak SNP detected by GWAS, we color-coded the individual accessions by genotype and plotted the origin of individual accessions on a world map (Fig. 3F). The results showed that the awnless accessions with a Ref-type allele (BTx623 type) were particularly abundant in south Africa and North America, while the awned accessions with an Alt-type allele (NOG type) were more common in Asia, North Africa, and Europe. Whether such geographic distribution reflects adaptation to regional climates or the result of human selection corresponding to the purpose of use is unclear.

\section{The candidate of the Dominant Awn Inhibitor gene in sorghum}

We compared the reference genome sequence of BTx623 with those from NOG that were determined by our re-sequencing data (Accession number: DRA008159). Consequently, a region of approximately $5 \mathrm{~kb}$ containing a single gene, Sobic003G421300, was shown to be missing in NOG (Supplementary Fig. 2A). Indeed, genomic PCR and Sanger sequencing verified this deletion in NOG that extended by $5.46 \mathrm{~kb}$ (Supplementary Fig. 2B and 2C, Fig. 4A). The peak SNP of the GWAS was located approximately $5.7 \mathrm{~kb}$ upstream from the transcriptional start site of Sobic003G421300. According to the Phytozome database (Goodstein et al. 2012), Sobic003G421300 encodes a protein of unknown function that contains an ALOG (Arabidopsis LSH1 and Oryza G1) domain (Fig. 4B). Among the ALOG family proteins in grass, G1, TAW1, and TH1/BLS1/AFD1 in rice have been well studied. LONG STERILE LEMMA1 (G1) is thought to be involved in transcriptional regulation and is expressed in the sterile lemma primordia to prevent its development in the wild-type rice plant; the sterile lemma is abnormally enlarged like the lemma in the $g l$ loss-of-function mutant (Yoshida et al. 2009). TAWAWA1 (TAW1), which is expressed in the inflorescence meristem (IM) and branch meristem, is also considered a transcription factor. TAW1 regulates the inflorescence architecture of rice by promoting IM activity and suppressing phase changes to spikelet meristem identity (Yoshida et al. 2013). For TRIANGULAR HULL1/BEAK LIKE SPIKELET1/ABNORMAL FLOWER AND DWARF1 (TH1/BLS1/ADF1), mutants showed various pleiotropic defects containing abnormal floral organs, and some results indicated that TH1/BLS1/ADF1 may influence cell sizes and cell numbers by negatively regulating the expression of cell proliferation and expansion related genes (Li et al. 2012, Ma et al. 2013, Sato et al. 2014, Ren et al. 2016, Peng et al. 2017).

These studies imply that ALOG family proteins inhibit the differentiation or development of specific organs. Taking these into account, it seemed plausible that Sobic003G421300 represents $q A L 3 / q A P 3$. We named this gene Dominant Awn Inhibitor (DAI). Given ALOG family proteins acting as transcription factors, we observed the subcellular localization of DAI in order to examine whether DAI also has transcription factor-like properties. We transiently expressed green fluorescent protein (GFP) or DAI-GFP fusion protein driven by the cauliflower mosaic virus $35 \mathrm{~S}$ promoter into onion epidermal cells by particle bombardment. While control GFP appeared to be localized to the cytoplasm and nucleus, but when magnified, GFP was present outside the nucleus, indicating that it was not actively localized to the nucleus (Fig. 4C upper). In contrast, the DAI-GFP 
fusion protein was exclusively localized in the nucleus (Fig. 4C bottom). Most likely, DAI is the transcription factor negatively regulating awn formation.

\section{Confirmation by further genotyping and complementation analysis}

Next, we performed additional experiments to verify that the DAI corresponded to $q A L 3 / q A P 3$. First, the link between the awned phenotype and $D A I$ deletion was carefully examined. While the peak SNP correlated well with awn phenotypes in most of the accessions (Fig. 3E), a few accessions showed discrepant phenotypes, including the awned ones with the Ref-type allele, and the awnless ones with the Alt-type allele. Therefore, we selected some accessions and examined whether they have the 5.46-kb fragment containing DAI (the DAI fragment) and/or whether they have additional mutations in DAI by Sanger sequencing. The peak genotype and the presence of the DAI fragment matched completely, for the accessions whose awn phenotypes were consistent with the peak genotype (Fig. 4D, 10 accessions from the top). For accessions with the discrepancy between the peak genotype and the awn phenotype, our experimental reassessment clarified that three accessions (PI 656029, PI 152771, and IS 29606) had consistency between the awn phenotype and the DAI insertion. In contrast, IS 24453 was remarkably different because it was awned despite having the DAI insertion. However, the sequence analysis of DAI in IS 24453 revealed that a single nucleotide substitution occurred (82 bp from the translation start point; C to T), resulting in a premature stop codon truncating DAI to only 28 aa (282 aa in wild-type), which was most likely a knockout. Thus, IS 24453 has the DAI fragment; however, DAI has been mutated and lost its function. These results strongly suggested that $D A I$ determines the presence of awn in sorghum.

Subsequently, we performed complementation analysis in sorghum using Agrobacterium-mediated transformation through tissue culture derived from immature embryos. We cloned the genomic fragment of BTx623 containing the putative promoter region (approximately $6.2 \mathrm{~kb}$ ) and $D A I$ into a binary vector (DAIpro::DAI) (Fig. 4A bottom). RIL232 is one of the awned lines in our RIL population and was used as a recipient. The obtained transformant $\left(\mathrm{T}_{0}\right)$ was found to have a single insertion based on the segregation ratio of $\mathrm{T}_{1}$ progeny. Genotyping of $\mathrm{T}_{1}$ plants were performed to distinguish homozygous (DAI/DAI), heterozygous (DAI/-), or azygous (-/-) individuals by qPCR. Five $\mathrm{T}_{1}$ plants of each genotype were selected for the observation of spikelets and measurement of AL. We found that homozygous plants completely lost their awn, heterozygous plants were partially awned, but mostly lost their awn, and azygous plants had the same level of awn as that in the parent (Fig.4 E, F). Together, these results led us to conclude that DAI is indeed the gene responsible for qAL3/qAP3.

\section{DAI may have originated from a gene duplication}

$D A I$ is present in the $5.64 \mathrm{~kb}$ genomic fragment that does not exist in accessions such as NOG. We questioned whether this fragment is evolutionarily derived from the deletion in the NOG ancestor or the insertion of the BTx623 ancestor. Somewhat surprisingly, the BLASTP search revealed the presence of one sorghum protein encoded by Sobic.010G223100 on chromosome 10, which showed 100\% identity with DAI in the amino acid sequence. Comparison of the coding sequences (CDSs) between the two genes revealed only one 
synonymous substitution at nucleotide 603-bp downstream from the translation start point (Supplementary Fig. 3). Moreover, DAI and Sobic.010G223100 showed significant homology not only between CDSs but also in the regions encompassing $5.15 \mathrm{~kb}$ (Supplementary Fig. 3). Given that Sobic.010G223100 is also present in the NOG genome (not shown) and that the awned predates the awnless phenotype during varietal differentiation, it is likely that the 5.46-kb fragment on chromosome 3 resulted from a duplication of Sobic.010G223100 in the BTx623 ancestor, rather than a deletion in the NOG ancestor. Therefore, we named this DAI homolog on chromosome $10 D A I^{\text {ori }}$. To further verify this hypothesis, synteny analysis was performed to compare the corresponding chromosomal regions among three other grass species (rice, brachypodium, and maize). There were syntenic blocks, which corresponded to the region around $D A I$ on chromosome 3 of sorghum, rice chromosome 1, brachypodium chromosome 2, and maize chromosomes 3 and 8 . However, there were no DAI homologs in their syntenic blocks (Fig. 5A and B upper). In contrast, $D A I^{\text {ori }}$ orthologs were found in syntenic blocks of all these grass species (in rice chromosome 6 , brachypodium chromosome 1 , and maize chromosomes 6 and 9) (Fig. 5A and B bottom). These observations support the hypothesis that $D A I^{\text {ri i }}$ is the origin of $D A I$ and might have jumped into chromosome 3 from chromosome 10 in the BTx623 ancestor.

Next, we performed BLASTP analysis on four grass species, including sorghum, using DAI/DAI ${ }^{\text {ori }}$ protein as a query, and constructed a phylogenetic tree using the selected related proteins. As a result, all proteins were grouped into clades represented by each rice G1 family protein (G1, G1L1-L9), although gene duplication was particularly prominent in maize (Fig. 5C). These ALOG family proteins were well-conserved, at least among these four species. DAI ${ }^{\text {ori }}$ was found to belong to the G1L2 clade of unknown function, which was different from TAW1 (G1L5 clade), TH1/BLS1/ADF1 (G1L6 clade), and G1 clade, whose functions have been reported. Functional analysis of genes in the G1L2 clade, including DAI ${ }^{\text {ori }}$, is a topic for future research.

\section{SbDAI can also function as an awn inhibitor in rice}

As a result of sequence comparison between the surrounding regions of $D A I$ and $D A I^{\text {ori }}$, we predicted how $D A I$ was generated by gene duplication through genome rearrangement (Supplementary Fig. 3, Fig. 5D). First, a fragment of approximately $5.15 \mathrm{~kb}$ encompassing the intron and CDS of $D A I^{\text {ori }}$ on chromosome 10 was duplicated and inserted into chromosome 3 (at approximately $72.6 \mathrm{Mb}$ ). A total of $80 \mathrm{bp}$ of unidentified sequence seemed to be added to both ends during the insertion; the origin of this sequence was currently unclear, because BLAST searches against all species did not find any hits other than its own. Subsequently, Tourist1b, a transposable element, was inserted into the intron region, and the gene might have undergone further rearrangement in the UTRs and introns, resulting in the birth of the DAI gene in its present form. There is no obvious evidence such as transposable elements at either end of the $5.46 \mathrm{~kb}$ inserted fragment.

The acquisition of DAI as a result of gene duplication, along with DAI as a conserved ALOG family protein among grass species, raised the possibility that the whole $D A I$ allele, including its promoter, may act as an awn inhibitor in species other than sorghum. To examine this possibility, we generated transformants using the awned rice cultivar Kasalath as a recipient, heterologously expressed $D A I$, and observed their spikelets. Using the pHGWFS7 vector as a backbone, the construct SbDAI was constructed that harbored the genomic region from BTx623, as shown in Figure 6A. As a negative control, the corresponding region from NOG was 
also cloned in the construct gNOG (Fig. 6A). Each binary vector carrying SbDAI or gNOG was transformed into Kasalath. Consequently, the individuals with gNOG exhibited an awned phenotype similar to Kasalath, while the individuals with SbDAI showed a complete awnless phenotype (Fig. 6 B-D). Three independent transformants $\left(\mathrm{T}_{0}\right)$ obtained for each construct were found to be single-copy transformants, and we determined whether the individual had transgene(s) (+ in Fig. 6E) or not (azygous: - in Fig. 6E) by PCR using DNA isolated from each of the segregated $\mathrm{T}_{1}$ populations. $\mathrm{A} \mathrm{T}_{1}$ plant for each genotype was selected for the measurement of $\mathrm{AL}$. The results of AL measurement showed that the awns of individuals with the SbDAI transgene were significantly shorter (almost completely zero) than those of their azygous (Fig. 6E). This indicated that SbDAI (native promoter $+D A I$ ) could also inhibit awn elongation in heterologous rice plants, supporting the gain-of-function of DAI function by gene duplication.

\section{Discussion}

In this study, three QTLs were identified on chromosomes 3 (qAL3/qAP3, Chr03:7192302572847396), 7 (qAL7, Chr07:58230302-Chr07:60603839), and 9 ( $q A L 9$, Chr09:56467317-57917278). Comparing these QTLs with those at the QTL Atlas (Mace et al. 2019), qAL3/qAP3 was likely to map to the awn length or presence-related QTLs reported by previous works (Boivin et al. 1999, Tao et al. 2000, Hart et al. 2001, Mace and Jordan 2010, Girma et al. 2019, Zhang et al. 2019). In this study, we corroborated this locus on chromosome 3 and identified $D A I$ as the dominant factor that inhibits awn formation.

In contrast to $q A L 3 / q A P 3$, no QTLs related to AL have been previously reported around $q A L 7$ and $q A L 9$. Investigation of possible genes corresponding to these QTLs identified two genes, Dwarfl (Dw1) and Dwarf3 (Dw3), which are the major plant height-responsible genes in sorghum. Dw1 (Sobic.009G230800, Chr09:57093313-57095643), which encodes a positive regulator gene of brassinosteroid signaling, regulates the length of internodes by controlling cell proliferation (Hilley et al. 2016, Yamaguchi et al. 2016, Hirano et al. 2017). Dw3 (Sobic.007G163800, Chr07:59821905-59829910) encodes an ABCB1 auxin efflux transporter (Multani et al. 2003). Previously, we detected $q P H 9$ (Chr09:56.57-57.68 Mb) and $q P H 7$ (Chr07:59.41-60.00 $\mathrm{Mb})$ as dominant plant height QTLs using the same RILs. We demonstrated that $D w 1$ and $D w 3$ are the genes responsible for $q P H 9$ and $q P H 7$ by sequencing analysis, respectively, highlighting that NOG has functional alleles for both genes (Kajiya-Kanegae et al. 2020). Given that $q A L 7$ (Chr07:58.23-60.60 Mb) and $q A L 9$ (Chr09:56.47-57.92 Mb) in this study contained $q P H 7$ and $q P H 9$ with high accuracy, $q A L 7$ and $q A L 9$ were thought to detect the same locus as $q P H 7$ and $q P H 9$, respectively. These two loci were also detected as significant QTLs for the length of spikelet-related organs (e.g., sessile spikelet, pedicel, anther, and style) using the same RILs (Takanashi et al. 2021). Based on the functional aspect of $D w 1$ and $D w 3$ (e.g., controlling organ size) and the direction of the allelic effects, in which the length of awn and spikelet-related organs and plant height were increased in the NOG allele, $q A L 7$ and $q A L 9$ also likely corresponded to $D w 3$ and $D w 1$, respectively.

A line of evidence including NILs, detailed genotyping, and complementation analysis using sorghum and rice, led us to conclude that $D A I$ is the gene responsible for $q A L 3 / q A P 3$, which determines the presence of 
awn in sorghum dominantly. The fact that most awnless accessions are likely to have achieved the awnless phenotype using DAI (Fig. 3 and 4) strongly suggests that DAI is an important gene for the development of modern, awnless sorghum varieties. DAI is useful and easy to manipulate genetically as a dominant factor, for example, in generating awnless $\mathrm{F}_{1}$ hybrids. It is therefore plausible that $D A I$ has been widely selected during the course of varietal diversification. DAI belongs to the ALOG family and is different from any other awn-regulating genes that have been reported in other grasses. It is noteworthy that wheat $B 1$, which encodes a $\mathrm{C} 2 \mathrm{H} 2$ zinc finger protein, functions similarly in inhibiting awn formation (DeWitt et al. 2020, Huang et al. 2020). The identification of $D A I$ in this study along with $B I$ reinforces the occurrence of such awn inhibitors, likely the transcription factors repressing cell elongation and/or organ formation in cereal crop species. However, it is noteworthy that they encode different transcription factors. Whether DAI is a gene associated with sorghum domestication is still unknown and needs to be verified by comparing genomic sequences of many accessions in the future. Future studies are required to understand the mechanism by which the putative transcription factor DAI inhibits awns by affecting downstream target genes.

Our finding that $D A I$ was born by gene duplication provides an interesting example of gain-of-function associated with panicle morphologies that are supplemental to plant growth. In this scenario, landing a duplicated gene into a region conferring proper expression by the novel promoter may be critically important to gain new functions. Moreover, we demonstrated in this study that the DAI genomic region containing its promoter can act as an awn inhibitor in rice, in which inhibitor genes have not been identified so far. Together, these results suggest that the rise of dominant awn inhibitors depends on such rare events during the diversification of cereals and is thus species-specific. In the case of DAI, the insertion site was within a non-coding DNA region that is $15.48 \mathrm{~kb}$ and $10.26 \mathrm{~kb}$ away from the neighboring gene both upstream (Sobic.003G421201) and downstream (Sobic.003G421400), respectively (Supplementary Fig. 2D). The upstream sequence of the insertion site in chromosome 3 was able to function as a promoter for expression in the awn or its primordium, which seems to be the reason why the $D A I^{\text {ori }}$ paralogue was able to function as a dominant awn inhibitor, although it is still unclear which sequences were essential promoter sequences for the function of DAI. Because there was a putative long interspersed nucleotide element (LINE, Fig. 4A) upstream of the insertion site, whose epigenetic modifications have been known to lead to changes in the expression of nearby genes (Ong-Abdullah et al. 2015), it is possible that this LINE was involved in the establishment of the $D A I$ function. Further elucidation of the mechanism of transcriptional regulation of $D A I$ is necessary in future studies

Phylogenetic analysis showed that DAI ${ }^{\text {ori }}$ belongs to the G1L2 clade. Although the function of the G1L2 group is unknown, the G1L2 subfamily including DAI ${ }^{\text {ori }}$ may have a similar function to suppress the differentiation or development of specific tissues or organs common to these grasses, because the putative common ancestral species must have a G1L2-like gene before the differentiation of these species. Since the ancestral grasses most likely had long awns, it is unlikely that ancestors of the G1L2 group proteins had the ability to suppress awn development. The reason for this hypothesis is that the locus around G1L2 has not been detected in a vast number of researches of rice awn that have been performed. Currently, we expect that DAI is a novel gene that has acquired a new function to inhibit awn by inserting a copy of $D A I^{\text {ori }}$, which has originally 
been unrelated to the regulation of awn, into chromosome 3 through gene duplication, resulting in the acquisition of a new promoter leading expression around the lemma or awn.

\section{Conclusion}

In this study, we identified the dominant awn inhibitor gene in sorghum, which has not been identified for a century since its first mention (Vinall and Cron 1921). We showed that DAI encodes a protein of unknown function with an ALOG domain, and it can almost eliminate the sorghum awn even in the heterologous state. Experiments using sorghum germplasm suggested that $D A I$ has been widely used in the process of modification or breeding of sorghum, perhaps because of its effectiveness and convenience.

$D A I$ is expected to be a very powerful and useful tool because an awnless phenotype can be achieved by only a single gene, and even in the heterozygous condition. For example, in the case of $F_{1}$ breeding by utilizing hybrid vigor, a high grain yield and awnless phenotype can be achieved by simply introducing DAI into either parent. As shown using rice, $D A I$ is likely to be effective on heterologous grass species, which makes it promising for future use. Further analysis of the DAI gene, which is different from any other gene identified in other grass species, will shed light in a better understanding of the mechanisms underlying the awn regulation and for the birth of a novel gene via gene duplication.

\section{Note to be added}

In the course of preparing this manuscript, Zhou et al. (2021) reported a gene named awnl, identical to $D A I$ in this study, as a candidate for an awn repressor in sorghum. Although their study did not provide direct evidence that awnl/DAI is a gene responsible for the dominant awn inhibitor, they provided several important results, including RNA sequencing and DNA affinity purification sequencing to identify the genes downstream of awnl. We believe that combining their report with ours will enrich our knowledge of awn regulation in sorghum. 


\section{Materials and methods}

\section{Plant materials}

The recombinant inbred line (RIL) population, derived from a parental cross between BTx623 and the Japanese landrace NOG was established as described in our previous study (Kajiya-Kanegae et al. 2020). In total, 245 RILs were generated by recurrent selfing of progeny derived from a cross with BTx623 and NOG at the Institute of Plant Science and Resources (IPSR) at Okayama University, as previously reported (Kajiya-Kanegae et al. 2020). A total of 289 accessions of sorghum germplasm were obtained from public bioresources (ICRISAT sorghum collection, NARO Genebank, and USDA). Supplementary Table 1 lists these accessions. Seeds of the rice cultivar, Kasalath, were kindly provided by Dr. Yasuo Nagato of the University of Tokyo. Near isogenic lines (NILs) were obtained starting from RIL205 (awnless), one of the heterozygous lines at the genotype of the marker associated with $q A L 3 / q A P 3$, in the RILs at the $\mathrm{F}_{6}$ generation (Fig. 2A). We established NILs ( $\mathrm{F}_{9}$ generation) through PCR genotyping using insertion-deletion polymorphisms for recurrent selfed progeny of RIL205 at IPSR (primers used for genotyping are listed in Supplementary Table 2).

\section{Genotype data}

Genotype data of the RILs were obtained using the restriction site-associated DNA sequencing (RAD-seq) method. Detailed methods and information for DNA extraction and library construction for the RAD-seq have been described in our previous report (Kobayashi et al. 2017). The RAD-seq reads of the 245 RILs from the $\mathrm{F}_{6}$ generation were mapped onto the reference genome Sorghum bicolor v2.1 (Paterson et al. 2009) using the bwa mem ( $\mathrm{Li}$ and Durbin 2010) algorithm with the default options, and then the marker positions were corrected to the information of the reference genome Sorghum bicolor v3.1.1 (McCormick et al. 2018). The SNPs were called with GATK (McKenna et al. 2010) and filtered with SAMtools (Li et al. 2009) under the following conditions: min-depth 5 , max-depth 30, max-missing 0.8 , minimum allele frequency 0.1 , remove-indels, min-alleles 2, max-alleles 2, and minQ 20. For segregation distortion analysis, we used a chi-squared test to calculate the deviation from the expected ratio (1:1) for each marker. All genotypes (BTx623/BTx623, NOG/NOG, BTx623/NOG) were used to elucidate allelic effects of QTLs in the $\mathrm{F}_{6}$ generation. To construct the genetic map for the RILs, only homozygous SNPs were selected, then SNPs with missing rates greater than $20 \%$ and duplicated markers were removed. In total, 1,792 high-quality SNPs were selected to construct the linkage map for QTL analysis. The map covered a total length of $682.4 \mathrm{cM}$ and had an average marker density of $0.4 \mathrm{cM}$.

Genotype data of sorghum germplasm were also obtained using RAD-seq. Detailed methods and information for genotyping have been described in our previous report (Yamazaki et al. 2020). SNPs with minor allele frequencies (1\%) or missing rates greater than $80 \%$ were filtered out for the GWAS study. Imputation was conducted using Beagle 5.0, with default parameter settings (Browning et al. 2018). In total, we obtained data for 50,678 SNP markers for 298 accessions. 


\section{Cultivation}

Field trials were performed in an open-air greenhouse at the experimental farm of IPSR (latitude: $34^{\circ} 35^{\prime} 31^{\prime}$ 'N, longitude: $133^{\circ} 46^{\prime} 7^{\prime}$ ' E), Kurashiki City, Okayama, Japan from June to September 2014 for the $F_{6}$ generation of the RILs. Plants were germinated in small trays filled with vermiculite for approximately 10-14 days, and then transplanted to either 30-cm diameter pots filled with soil from the IPSR field ( $\mathrm{n}=1$ per line).

Field trials were performed in a green field at the University of Tokyo (latitude: $35^{\circ} 42^{\prime} 58.5^{\prime}$ ' $\mathrm{N}$, longitude: 139 $45^{\prime} 44.5^{\prime \prime}$ E), Yayoi, Bunkyo-Ku, Tokyo, Japan, from May to October in 2015, 2019, and 2020. Plants were germinated in 200-cell trays filled with synthetic culture soil (BONSOL 1; Sumitomo Chemical Co., Ltd., Japan) for two weeks, and then transplanted into the field $(n=2$ per line). The space between ridges was 60 $\mathrm{cm}$, with a $15 \mathrm{~cm}$ distance between individuals in the same ridge. The field was fertilized using $\mathrm{N}: \mathrm{P}: \mathrm{K}=10: 10: 10\left(\mathrm{~kg} \mathrm{ha}^{-1}\right)$ fertilizer before the trials in each year. Cultivation and phenotyping of awn-related traits for RILs were conducted in 2015 using the $\mathrm{F}_{7}$ generation, and those for germplasm were conducted in 2015, 2019, and 2020.

Transgenic sorghum and rice plants were grown and maintained in a biohazard greenhouse at $30{ }^{\circ} \mathrm{C}$ (day) and $25^{\circ} \mathrm{C}$ (night) under natural light conditions.

\section{Phenotyping for QTL analysis and GWAS}

To standardize developmental stages of spikelets among the RILs and germplasm, we sampled spikelets at the just-before-flowering stage in each line without the $\mathrm{F}_{6}$ generation of the RILs cultivated at Okayama, which were sampled during the grain-filling stage. In the inflorescence that began flowering at the top, we defined spikelets at the just-before-flowering stage (spikelets that would flower within a day) as those attached to the primary branch just below the flowered primary branch. For each line, we picked awns from five spikelets sampled from the top, middle, and bottom of the panicle, mounted them on a paper board, and photographed with a digital camera (OM-D E-M5; Olympus, Japan). Awn length (AL) was measured using ImageJ $1.49 \mathrm{v}$ (Schneider et al. 2012). We also scored awn presence values on a two-step scale, with awnless lines as 0 and awned lines as 1 . For all awn-related traits, the average value of five independent awns was used as the phenotypic value of an individual plant.

\section{QTL analysis}

We performed QTL analysis for awn-related traits in the RILs (AL; AP, awn presence; and AL in awned lines only). Genotype probabilities were calculated using the calc. genoprobility function with a step size of $1 \mathrm{cM}$ and an assumed genotyping error probability of 0.05, using the Kosambi map function (Kosambi 1943) as implemented in the R (R Core Team 2021)/qtl package (Broman et al. 2003). QTL analysis was performed using the composite interval mapping (CIM) function of the R/qtl package with the Haley-Knott regression method (Haley and Knott 1992). Linkage analysis was performed using the R/qtl package in R version 4.1.0. The LOD significance threshold for detecting QTLs was calculated by performing 1,000 iterations using the R/qtl permutation test. Confidence intervals for QTLs were estimated based on the 2.0-LOD support interval, and the nearest flanking markers located outside the boundary of each confidence interval were defined as both 
ends of the marker intervals. The additive effect and the percentage of phenotypic variance explained (PVE) by each QTL were obtained using the fitqtl function of the R/qtl package. Box plots were created using the R/ggplot2 package (Wickham 2016).

\section{GWAS}

We performed GWAS for awn-related traits in germplasm (AL and AP). GWAS was performed using a linear mixed model implemented by the "association.test" function in the gaston package ver. 1.5.7 in $\mathrm{R}$ (Perdry et al. 2020). A genetic relationship matrix specifying a random additive effect was computed using the 'A.mat' function of the rrBLUP package (Endelman 2011). The P values of the marker-trait associations were calculated using the Wald test. The genome-wide significance threshold was obtained based on the BenjaminiHochberg false discovery rate [FDR; Benjamini and Hochberg (1995)] at a 0.1\% level. The LD heatmaps surrounding peaks in the GWAS were constructed using the "LD" function of the gaston package. To determine the geographic distribution of alleles at the peak SNP in GWAS, the world map layout was generated using the ggplot2 package in R. Passport data of accessions were obtained from the U.S. National Plant Germplasm System (https://npgsweb.ars-grin.gov/gringlobal/search), the NARO Genebank (https://www.gene.affrc.go.jp/databases-core_collections_ws.php), and the ICRISAT sorghum collection (http://www.icrisat.org/what-we-do/crops/sorghum/Project1/Sorghum_minicore.htm).

\section{Genotyping of the $5.46 \mathrm{~kb}$ fragment containing DAI for BTx623, NOG, and selected germplasm}

Healthy young leaves from each line were collected, and genomic DNA was extracted using a Maxwell RSC Plant DNA Kit (Promega, USA). The presence or absence of the $5.46 \mathrm{~kb}$ fragment containing DAI was confirmed by PCR (primers 1 and 2), and its sequence information was determined by direct Sanger sequencing using the same primers.

\section{Plasmid construction}

To express GFP and DAI-GFP transiently, we made the corresponding constructs using the Gateway cloning technology (Invitrogen /Thermo Fisher Scientific, USA). The CDS of DAI without a stop codon was amplified by PCR using BTx623 DNA as a template (primers 3 and 4), and the amplified DNA fragment and an annealed linker oligo (for GFP: primers 5 and 6) was introduced into the pENTR/D-TOPO vector (Invitrogen; sequences were confirmed by Sanger sequencing using primers 7 and 8) and then subcloned into a pH7FWG2 vector (Karimi et al. 2002) using LR clonase (Invitrogen) to obtain 35Spro::DAI-GFP and 35Spro::GFP constructs.

For sorghum and Kasalath transgenic lines, the constructs were made using the In-Fusion HD cloning technology (Clontech/Takara Bio, Japan). For DAIpro::DAI, the pBUH3 vector (Nigorikawa et al. 2012) was linearized by PCR (primers 9 and 10). The DNA fragment containing DAI native promoter and the CDS of DAI with a stop codon were amplified by PCR using the SbDAI plasmid described below as a template (primers 11 and 12), and the PCR-amplified DNA fragment was cloned into linearized pBUH3 via In-Fusion HD cloning. The sequence of the DAIpro::DAI plasmid was confirmed by Sanger sequencing using primers 13 to 18 . For 
SbDAI, the DNA fragment containing DAI native promoter and the CDS of DAI with a stop codon were amplified by nested PCR using BTx623 DNA as a template (primers 25-28). For gNOG, the DNA fragment was amplified by nested PCR using NOG DNA as a template (primers 25, 2, 27, and 29). Amplified DAIpro::DAI and gNOG fragments were cloned into the NcoI/SacI-digested pHGWFS7 vector (Karimi et al. 2002) via In-Fusion HD to generate SbDAI and gNOG plasmids, respectively. The sequence of these plasmids was confirmed by Sanger sequencing using primers 16 and 30 to 33 .

\section{Particle bombardment}

GFP and DAI-GFP plasmids were transiently introduced into onion epidermal cells using a helium-driven particle accelerator (PDS/1000; Bio-Rad Laboratories, USA). Particle bombardment experiments were performed according to the conditions described by Arimura et al. (2004). Before microscopic observation, onion epidermal cells were treated with distilled water containing $2 \mathrm{mg} \mathrm{L}^{-1}$ 4',6-diamidino-2-phenylindole dihydrochloride (DAPI) to stain nuclei.

\section{Microscopic observations}

Enlarged images of secondary branches and spikelets of sorghum and Kasalath were captured under a stereomicroscope (M125; Leica, Germany) with a CCD camera (MC170 HD; Leica). Onion epidermal cells expressing GFP and DAI-GFP under control of the 35S promoter were observed using a Leica STELLARIS5 confocal microscope (Leica) with a HC PL APO CS2 20× DRY objective lens (N.A. $=0.75$ ), HC PL APO CS2 $63 \times$ OIL objective lens (N.A. $=1.40)$, and HyD detectors. The Leica STELLARIS5 microscope was controlled by LAS X software (Leica), DAPI and GFP were excited with a $405 \mathrm{~nm}$ DMDO laser and a $495 \mathrm{~nm}$ tuned white light laser, respectively.

\section{Agrobacterium-mediated transformation}

Agrobacterium-mediated sorghum transformation of RIL232 (one of the lines in our RILs, awned) was performed according to $\mathrm{Wu}$ et al. (2014) with several modifications. Immature embryos isolated from grains 15 days after pollination were pre-cultured in DBC medium at $25^{\circ} \mathrm{C}$ in the dark. Pre-cultured immature embryos were collected in infection medium, pre-treated at $43{ }^{\circ} \mathrm{C}$ for $15 \mathrm{~min}$, and centrifuged $(20,000 \times \mathrm{g}$ for 10 min). Pre-treated immature embryos were inoculated with Agrobacterium tumefaciens strain EHA105 harboring the DAIpro::DAI vector and co-cultured at $25{ }^{\circ} \mathrm{C}$ for 5 days in the dark. After 5 days of resting culture, calli were selected on a medium containing $15-20 \mathrm{mg} \mathrm{L}^{-1}$ hygromycin, and resistant calli were transferred to regeneration medium containing $10 \mathrm{mg} \mathrm{L}^{-1}$ hygromycin. Regenerated shoots were cultured on rooting medium until the roots were produced. We obtained several plantlets, but only one was confirmed as a transformant by PCR. Segregation analyses of $\mathrm{T}_{1}$ plants derived from the self-pollinated $\mathrm{T}_{0}$ plant by PCR using primers 19 and 20 revealed that the $\mathrm{T}_{0}$ plant was heterozygous for a single copy of the transgene (segregation ratio was approximately 3:1). We confirmed the number of transgenes in segregated $\mathrm{T}_{1}$ plants by qPCR using primers 21 to 24 and selected five individuals each as homozygous, heterozygous, and azygous for the measurement of AL. For each line, we picked up awns from 30 spikelets sampled from the middle portion of the panicle at the 
just-before-flowering stage and measured the $\mathrm{AL}$ as described above.

The constructed gNOG and SbDAI vectors were transformed into calli induced from the scutellum of wild type rice seeds (cv. Kasalath, awned) by Agrobacterium tumefaciens (strain EHA105), according to Toki et al. (2006). Transgenic plants were selected on medium containing $50 \mathrm{mg} \mathrm{L}^{-1}$ hygromycin. Hygromycin-resistant plants $\left(\mathrm{T}_{0}\right)$ were transplanted into soil and grown at $30^{\circ} \mathrm{C}$ (day) and $25{ }^{\circ} \mathrm{C}$ (night). Genotyping of $\mathrm{T}_{0}$ plants was performed by PCR using primers 19, 34, and 35, and we obtained three independent transgenic lines for each construct. $\mathrm{T}_{1}$ plants derived from the self-pollinated $\mathrm{T}_{0}$ plants were genotyped by PCR, and individuals with/without transgene(s) were selected for the measurement of AL. For each line, we sampled panicles before flowering and measured the AL of apical spikelets on each primary and secondary branch (approximately 100 spikelets).

\section{Synteny analysis}

Synteny analysis was performed using the online SynMap2 software (Haug-Baltzell et al. 2017). Datasets were chosen for dot plots as follows: Sorghum bicolor (id331), Oryza sativa (id3), Brachypodium distachyon (id39836), and Zea mays (id38215). Only the results of chromosomes corresponding to DAI (Sb_Chr03) and $D A I^{\text {ori }}$ (Sb_Chr10) were extracted from the output files of SynMap2 and dot plots were drawn using ggplot2/R. Enlarged images around $D A I$ or $D A I^{\text {ori }}$ in Figure $5 \mathrm{~B}$ were based on the reference genomes of Sorghum bicolor (v3.1.1), Oryza sativa (v7.0), Brachypodium distachyon (v3.1), and Zea mays (RefGen_V4) in Phytozome 13 (Goodstein et al. 2012).

\section{Phylogenetic analysis}

The amino acid sequence of Sobic.003G421300/DAI was used as the query sequence for a BLASTP search against the protein databases of Sorghum bicolor (v3.1.1), Oryza sativa (v7.0), Brachypodium distachyon (v3.1), and Zea mays (RefGen_V4) using Phytozome 13. Fifty matches with an expected value $<\mathrm{e}^{-40}$ were selected, and a phylogenetic tree was constructed using the ClustalW alignment and maximum likelihood methods based on the JTT matrix-based model (Jones et al. 1992) using default settings in the MEGA 7 package (Kumar et al. 2016). The following protein sequences from the Phytozome database were used for the phylogenetic analysis. S. bicolor: Sobic.001G219400, Sobic.002G029700, Sobic.003G342600, Sobic.003G421300 (DAI), Sobic.004G052800, Sobic.004G219600, Sobic.004G338400, Sobic.005G094266, Sobic.006G147500, Sobic.009G102600, Sobic.009G170100, and Sobic.010G225100 (DAI ${ }^{\text {ori }}$ ); O. sativa: LOC_Os01g61310 (G1L7), LOC_Os02g07030 (G1L1), LOC_Os02g41460 (G1L3), LOC_Os02g56610 (G1L6/TH1/BLS1/ADF1), LOC_Os04g43580 (G1L4), LOC_Os05g28040 (G1L9), LOC_Os05g39500 (G1L8), LOC_Os06g46030 (G1L2), LOC_Os07g04670 (G1), and LOC_Os10g33780 (G1L5/TAW1), B. distachyon: Bradi1g32445, Bradi1g58070, Bradi2g22561, Bradi2g29110, Bradi2g53880, Bradi3g04960, Bradi3g28800, Bradi3g49140, Bradi3g54800, and Bradi5g15700; Z. mays: Zm00001d003057, Zm00001d010507, Zm00001d010596, Zm00001d012473， Zm00001d014032， Zm00001d015342， Zm00001d017263, Zm00001d018433, Zm00001d018775, Zm00001d025863, Zm00001d032696, Zm00001d036617, Zm00001d038016, Zm00001d038442, Zm00001d043011, Zm00001d046998, Zm00001d051143, and 
Zm00001d051966.

\section{Data Availability}

The sequence data reported in this paper have been submitted to the DDBJ Sequence Read Archive with the accession number DRA008159.

\section{Author Contributions}

W.S. conceived the work dissecting sorghum awn development along with H.T.; material preparation, field experiments, and data analysis in QTL and GWAS was performed by H.T., H.K.-K., W.S., M.I. with supervision by M.K., K.Y., H.I., and N.T.; NIL was established by W.S and analyzed by H.T.; Sorghum transformation was performed by A.N., J.Y., and H.T. with supervision by N.T.; Rice transformation was performed by H.T.; final data were prepared for publication by H.T., H. K.-K., and W.S., and the manuscript was written by H.T. and W.S., on behalf of all authors.

\section{Funding}

This work is partly supported by the Core Research for Evolutional Science and Technology (CREST) from the Japan Science and Technology Agency (to N.T. and W.S.), KAKENHI grants (17H01457 to N.T., 18K19343 and 21H02597 to W.S., 18K05570 to H.T.) from the Japanese Society for the Promotion of Science (JSPS). We also thank the Oohara Foundation for financial support of our research group.

\section{Disclosures}

The authors have no conflicts of interest to declare.

\section{Acknowledgments}

We thank Tsuneaki Takami, Zihuan Jing, Fiona Wacera, Norikazu Ohnishi, Everlyne Omollo, and Rie Hijiya (IPSR, Okayama University) for their help with the cultivation at the IPSR, Kurashiki, Okayama. We are also grateful to the members of the Laboratory of Plant Molecular Genetics, and the Laboratory of Biometry and Bioinformatics at the University of Tokyo for their help with the cultivation in Tokyo. 


\section{References}

Amarasinghe, Y.P.J., Kuwata, R., Nishimura, A., Phan, P.D.T., Ishikawa, R. and Ishii, T. (2020) Evaluation of domestication loci associated with awnlessness in cultivated rice, Oryza sativa. Rice $(N Y)$ 13: 26.

Arimura, S., Yamamoto, J., Aida, G.P., Nakazono, M. and Tsutsumi, N. (2004) Frequent fusion and fission of plant mitochondria with unequal nucleoid distribution. Proc. Natl. Acad. Sci. U. S. A. 101: 7805-7808.

Benjamini, Y. and Hochberg, Y. (1995) Controlling the false discovery rate - A practical and powerful approach to multiple testing. J. R. Stat. Soc. B 57: 289-300.

Bessho-Uehara, K., Wang, D.R., Furuta, T., Minami, A., Nagai, K., Gamuyao, R., et al. (2016) Loss of function at RAE2, a previously unidentified EPFL, is required for awnlessness in cultivated Asian rice. Proc. Natl. Acad.

Sci. U. S. A. 113: 8969-8974.

Bessho-Uehara, K., Yamagata, Y., Takashi, T., Makino, T., Yasui, H., Yoshimura, A., et al. (2021) Exploring the loci responsible for awn development in rice through comparative analysis of all AA genome species. Plants (Basel) 10.

Boivin, K., Deu, M., Rami, J.F., Trouche, G. and Hamon, P. (1999) Towards a saturated sorghum map using RFLP and AFLP markers. Theor. Appl. Genet. 98: 320-328.

Broman, K.W., Wu, H., Sen, S. and Churchill, G.A. (2003) R/qtl: QTL mapping in experimental crosses. Bioinformatics 19: 889-890.

Browning, B.L., Zhou, Y. and Browning, S.R. (2018) A one-penny imputed genome from next-generation reference panels. Am. J. Hum. Genet. 103: 338-348.

Cavanagh, A.M., Morgan, J.W. and Godfree, R.C. (2020) Awn morphology influences dispersal, microsite selection and burial of Australian native grass diaspores. Front. Ecol. Evol. 8.

DeWitt, N., Guedira, M., Lauer, E., Sarinelli, M., Tyagi, P., Fu, D.L., et al. (2020) Sequence-based mapping identifies a candidate transcription repressor underlying awn suppression at the B1 locus in wheat. New Phytol. 225: 326-339.

Dockter, C., Gruszka, D., Braumann, I., Druka, A., Druka, I., Franckowiak, J., et al. (2014) Induced variations in brassinosteroid genes define barley height and sturdiness, and expand the green revolution genetic toolkit. Plant Physiol. 166: 1912-1927.

Elbaum, R., Zaltzman, L., Burgert, I. and Fratzl, P. (2007) The role of wheat awns in the seed dispersal unit. Science 316: 884-886.

Endelman, J.B. (2011) Ridge regression and other kernels for genomic selection with R package rrBLUP. Plant Genome-Us 4: 250-255.

Furuta, T., Komeda, N., Asano, K., Uehara, K., Gamuyao, R., Angeles-Shim, R.B., et al. (2015) Convergent loss of awn in two cultivated rice species Oryza sativa and Oryza glaberrima is caused by mutations in different loci. G3-Genes Genom Genet. (Bethesda) 5: 2267-2274.

Girma, G., Nida, H., Seyoum, A., Mekonen, M., Nega, A., Lule, D., et al. (2019) A large-scale genome-wide association analyses of Ethiopian sorghum landrace collection reveal loci associated with important traits. Front. Plant Sci. 10: 691.

Goodstein, D.M., Shu, S., Howson, R., Neupane, R., Hayes, R.D., Fazo, J., et al. (2012) Phytozome: A 
comparative platform for green plant genomics. Nucleic Acids Res. 40: D1178-D1186.

Grundbacher, F.J. (1963) The physiological function of the cereal awn. Bot. Rev. 29: 366-381.

Gu, B.G., Zhou, T.Y., Luo, J.H., Liu, H., Wang, Y.C., Shangguan, Y.Y., et al. (2015) An-2 encodes a cytokinin synthesis enzyme that regulates awn length and grain production in rice. Mol. Plant 8: 1635-1650.

Haley, C.S. and Knott, S.A. (1992) A simple regression method for mapping quantitative trait loci in line crosses using flanking markers. Heredity 69: 315-324.

Hart, G.E., Schertz, K.F., Peng, Y. and Syed, N.H. (2001) Genetic mapping of Sorghum bicolor (L.) Moench QTLs that control variation in tillering and other morphological characters. Theor. Appl. Genet. 103: 1232-1242. Haug-Baltzell, A., Stephens, S.A., Davey, S., Scheidegger, C.E. and Lyons, E. (2017) SynMap2 and SynMap3D: Web-based whole-genome synteny browsers. Bioinformatics 33: 2197-2198.

Hilley, J., Truong, S., Olson, S., Morishige, D. and Mullet, J. (2016) Identification of Dw1, a regulator of sorghum stem internode length. PLOS ONE 11: e0151271.

Hirano, K., Kawamura, M., Araki-Nakamura, S., Fujimoto, H., Ohmae-Shinohara, K., Yamaguchi, M., et al. (2017) Sorghum DW1 positively regulates brassinosteroid signaling by inhibiting the nuclear localization of BRASSINOSTEROID INSENSITIVE 2. Sci. Rep. 7: 126.

Hua, L., Wang, D.R., Tan, L.B., Fu, Y.C., Liu, F.X., Xiao, L.T., et al. (2015) LABA1, a domestication gene associated with long, barbed awns in wild rice. Plant Cell 27: 1875-1888.

Huang, D.Q., Zheng, Q., Melchkart, T., Bekkaoui, Y., Konkin, D.J.F., Kagale, S., et al. (2020) Dominant inhibition of awn development by a putative zinc-finger transcriptional repressor expressed at the B1 locus in wheat. New Phytol. 225: 340-355.

Jin, J., Hua, L., Zhu, Z.F., Tan, L.B., Zhao, X.H., Zhang, W.F., et al. (2016) GAD1 encodes a secreted peptide that regulates grain number, grain length, and awn development in rice domestication. Plant Cell 28:

2453-2463.

Jing, Z., Wacera, W.F., Takami, T., Takanashi, H., Fukada, F., Kawano, Y., et al. (2021) NB-LRR-encoding genes conferring susceptibility to organophosphate pesticides in sorghum. Sci. Rep. in press.

Jones, D.T., Taylor, W.R. and Thornton, J.M. (1992) The rapid generation of mutation data matrices from protein sequences. Comput. Appl. Biosci. 8: 275-282.

Kajiya-Kanegae, H., Takanashi, H., Fujimoto, M., Ishimori, M., Ohnishi, N., Wacera, W.F., et al. (2020) RAD-seq-based high-density linkage map construction and QTL mapping of biomass-related traits in sorghum using the Japanese landrace Takakibi NOG. Plant Cell Physiol. 61: 1262-1272.

Karimi, M., Inze, D. and Depicker, A. (2002) GATEWAY vectors for Agrobacterium-mediated plant transformation. Trends Plant Sci. 7: 193-195.

Kato, K., Miura, H., Akiyama, M., Kuroshima, M. and Sawada, S. (1998) RFLP mapping of the three major genes, Vrn1, Q and B1, on the long arm of chromosome 5A of wheat. Euphytica 101: 91-95.

Kjack, J.L. and Witters, R.E. (1974) Physiological-activity of awns in isolines of atlas barley. Crop Sci. 14: 243-248.

Kobayashi, M., Ohyanagi, H., Takanashi, H., Asano, S., Kudo, T., Kajiya-Kanegae, H., et al. (2017) Heap: A highly sensitive and accurate SNP detection tool for low-coverage high-throughput sequencing data. DNA Res. 
24: 397-405.

Kosambi, D.D. (1943) The estimation of map distances from recombination values. Ann Eugenic 12: 172-175. Kumar, S., Stecher, G. and Tamura, K. (2016) MEGA7: Molecular evolutionary genetics analysis version 7.0 for bigger datasets. Mol. Biol. Evol. 33: 1870-1874.

Li, H. and Durbin, R. (2010) Fast and accurate long-read alignment with Burrows-Wheeler transform. Bioinformatics 26: 589-595.

Li, H., Handsaker, B., Wysoker, A., Fennell, T., Ruan, J., Homer, N., et al. (2009) The Sequence Alignment/Map format and SAMtools. Bioinformatics 25: 2078-2079.

Li, X.J., Sun, L.J., Tan, L.B., Liu, F.X., Zhu, Z.F., Fu, Y.C., et al. (2012) TH1, a DUF640 domain-like gene controls lemma and palea development in rice. Plant Mol. Biol. 78: 351-359.

Li, X.J., Wang, H.G., Li, H.B., Zhang, L.Y., Teng, N.J., Lin, Q.Q., et al. (2006) Awns play a dominant role in carbohydrate production during the grain-filling stages in wheat (Triticum aestivum). Physiol. Plant. 127: 701-709.

Luo, J.H., Liu, H., Zhou, T.Y., Gu, B.G., Huang, X.H., Shangguan, Y.Y., et al. (2013) An-1 encodes a basic Helix-Loop-Helix protein that regulates awn development, grain size, and grain number in rice. Plant Cell 25: 3360-3376.

Ma, X.D., Cheng, Z.J., Wu, F.Q., Jin, M.N., Zhang, L.G., Zhou, F., et al. (2013) BEAK LIKE SPIKELET1 is required for lateral development of lemma and palea in rice. Plant Mol. Biol. Rep. 31: 98-108.

Mace, E., Innes, D., Hunt, C., Wang, X., Tao, Y., Baxter, J., et al. (2019) The Sorghum QTL Atlas: a powerful tool for trait dissection, comparative genomics and crop improvement. Theor. Appl. Genet. 132: $751-766$.

Mace, E.S. and Jordan, D.R. (2010) Location of major effect genes in sorghum (Sorghum bicolor (L.) Moench). Theor. Appl. Genet. 121: 1339-1356.

McCormick, R.F., Truong, S.K., Sreedasyam, A., Jenkins, J., Shu, S.Q., Sims, D., et al. (2018) The Sorghum bicolor reference genome: improved assembly, gene annotations, a transcriptome atlas, and signatures of genome organization. Plant J. 93: 338-354.

McKenna, A., Hanna, M., Banks, E., Sivachenko, A., Cibulskis, K., Kernytsky, A., et al. (2010) The Genome Analysis Toolkit: a MapReduce framework for analyzing next-generation DNA sequencing data. Genome Res. 20: $1297-1303$.

Motzo, R. and Giunta, F. (2002) Awnedness affects grain yield and kernel weight in near-isogenic lines of durum wheat. Aust. J. Agric. Res. 53: 1285-1293.

Multani, D.S., Briggs, S.P., Chamberlin, M.A., Blakeslee, J.J., Murphy, A.S. and Johal, G.S. (2003) Loss of an MDR transporter in compact stalks of maize br2 and sorghum dw3 mutants. Science 302: 81-84.

Nigorikawa, M., Watanabe, A., Furukawa, K., Sonoki, T. and Ito, Y. (2012) Enhanced saccharification of rice straw by overexpression of rice exo-glucanase. Rice 5: 14.

Ogbaga, C.C., Stepien, P. and Johnson, G.N. (2014) Sorghum (Sorghum bicolor) varieties adopt strongly contrasting strategies in response to drought. Physiol. Plant. 152: 389-401.

Ohnishi, N., Wacera, W.F. and Sakamoto, W. (2019) Photosynthetic responses to high temperature and strong light suggest potential post-flowering drought tolerance of sorghum Japanese landrace Takakibi. Plant Cell 
Physiol. 60: 2086-2099.

Ong-Abdullah, M., Ordway, J.M., Jiang, N., Ooi, S.E., Kok, S.Y., Sarpan, N., et al. (2015) Loss of Karma transposon methylation underlies the mantled somaclonal variant of oil palm. Nature 525: 533-537.

Paterson, A.H., Bowers, J.E., Bruggmann, R., Dubchak, I., Grimwood, J., Gundlach, H., et al. (2009) The Sorghum bicolor genome and the diversification of grasses. Nature 457: 551-556.

Peng, P., Liu, L.H., Fang, J.J., Zhao, J.F., Yuan, S.J. and Li, X.Y. (2017) The rice TRIANGULAR HULL1 protein acts as a transcriptional repressor in regulating lateral development of spikelet. Sci. Rep. $7: 13712$. Perdry, H., Dandine-Roulland, C., Bandyopadhyay, D. and Kettner, L. (2020) gaston: Genetic Data Handling (QC, GRM, LD, PCA) \& Linear Mixed Models. R package version 1.5.7.

Price, H.J., Dillon, S.L., Hodnett, G., Rooney, W.L., Ross, L. and Johnston, J.S. (2005) Genome evolution in the genus Sorghum (Poaceae). Ann. Bot. 95: 219-227.

R Core Team (2021) R: A language and environment for statistical computing. R Foundation for Statistical Computing, Vienna, Austria. URL https://www.R-project.org/.

Ren, D.Y., Rao, Y.C., Wu, L.W., Xu, Q.K., Li, Z.Z., Yu, H.P., et al. (2016) The pleiotropic ABNORMAL FLOWER AND DWARF1 affects plant height, floral development and grain yield in rice. J. Integr. Plant Biol. 58: 529-539.

Sato, D.S., Ohmori, Y., Nagashima, H., Toriba, T. and Hirano, H.Y. (2014) A role for TRIANGULAR HULL1 in fine-tuning spikelet morphogenesis in rice. Genes Genet. Syst. 89: 61-69.

Sato, S., Ishikawa, S., Shimono, M. and Shinjyo, C. (1996) Genetic studies on an awnness gene An-4 on chromosome 8 in rice, Oryza sativa L. Breed. Sci. 46: 321-327.

Schneider, C.A., Rasband, W.S. and Eliceiri, K.W. (2012) NIH Image to ImageJ: 25 years of image analysis. Nat. Methods 9: 671-675.

Shehzad, T., Iwata, H. and Okuno, K. (2009) Genome-wide association mapping of quantitative traits in sorghum (Sorghum bicolor (L.) Moench) by using multiple models. Breed. Sci.59: 217-227.

Sieglinger, J.B., Swanson, A.F. and Martin, J.H. (1934) Inheritance of awn development in Sorghums. J. Agric. Res. 49: 663-668.

Sourdille, P., Cadalen, T., Gay, G., Gill, B. and Bernard, M. (2002) Molecular and physical mapping of genes affecting awning in wheat. Plant Breed. 121: 320-324.

Takahashi, R. (1955) The origin and evolution of cultivated barley. Adv. Genet. 7: 227-266.

Takanashi, H., Shichijo, M., Sakamoto, L., Kajiya-Kanegae, H., Iwata, H., Sakamoto, W., et al. (2021) Genetic dissection of QTLs associated with spikelet-related traits and grain size in sorghum. Sci. Rep. 11: 9398.

Taketa, S., Yuo, T., Sakurai, Y., Miyake, S. and Ichii, M. (2011) Molecular mapping of the short awn 2 (lks2) and dense spike 1 (dsp1) genes on barley chromosome 7H. Breed. Sci. 61: 80-85.

Tao, Y.Z., Henzell, R.G., Jordan, D.R., Butler, D.G., Kelly, A.M. and McIntyre, C.L. (2000) Identification of genomic regions associated with stay green in sorghum by testing RILs in multiple environments. Theor. Appl. Genet. 100: 1225-1232.

Toki, S., Hara, N., Ono, K., Onodera, H., Tagiri, A., Oka, S., et al. (2006) Early infection of scutellum tissue with Agrobacterium allows high-speed transformation of rice. Plant J. 47: 969-976. 
Toriba, T. and Hirano, H.Y. (2014) The DROOPING LEAF and OsETTIN2 genes promote awn development in rice. Plant J. 77: 616-626.

Tuinstra, M.R., Grote, E.M., Goldsbrough, P.B. and Ejeta, G. (1997) Genetic analysis of post-flowering drought tolerance and components of grain development in Sorghum bicolor (L.) Moench. Mol. Breed. 3: 439-448.

Vinall, H.N. and Cron, A.B. (1921) Improvement of sorghums by hybridization. J. Hered. 12: 435-443.

Watkins, A.E. and Ellerton, S. (1940) Variation and genetics of the awn in Triticum. J. Genet. 40: 243-270.

Wickham, H. (2016) ggplot2: Elegant graphics for data analysis. Springer-Verlag New York.

Wu, E., Lenderts, B., Glassman, K., Berezowska-Kaniewska, M., Christensen, H., Asmus, T., et al. (2014)

Optimized Agrobacterium-mediated sorghum transformation protocol and molecular data of transgenic sorghum plants. In Vitro Cell. Dev. Biol. Plant 50: 9-18.

Yamaguchi, M., Fujimoto, H., Hirano, K., Araki-Nakamura, S., Ohmae-Shinohara, K., Fujii, A., et al. (2016)

Sorghum Dw1, an agronomically important gene for lodging resistance, encodes a novel protein involved in cell proliferation. Sci. Rep. 6: 28366.

Yamazaki, K., Ishimori, M., Kajiya-Kanegae, H., Takanashi, H., Fujimoto, M., Yoneda, J., et al. (2020) Effect of salt tolerance on biomass production in a large population of sorghum accessions. Breed. Sci. 70: 167-175.

Yano, K., Yamamoto, E., Aya, K., Takeuchi, H., Lo, P.C., Hu, L., et al. (2016) Genome-wide association study using whole-genome sequencing rapidly identifies new genes influencing agronomic traits in rice. Nat. Genet. 48: 927-934.

Yoshida, A., Sasao, M., Yasuno, N., Takagi, K., Daimon, Y., Chen, R.H., et al. (2013) TAWAWA1, a regulator of rice inflorescence architecture, functions through the suppression of meristem phase transition. Proc. Natl. Acad. Sci. U. S. A. 110: 767-772.

Yoshida, A., Suzaki, T., Tanaka, W. and Hirano, H.Y. (2009) The homeotic gene long sterile lemma (G1) specifies sterile lemma identity in the rice spikelet. Proc. Natl. Acad. Sci. U. S. A. 106: 20103-20108.

Yoshioka, M., Iehisa, J.C.M., Ohno, R., Kimura, T., Enoki, H., Nishimura, S., et al. (2017) Three dominant awnless genes in common wheat: Fine mapping, interaction and contribution to diversity in awn shape and length. PLOS ONE 12: e0176148.

Yuo, T., Yamashita, Y., Kanamori, H., Matsumoto, T., Lundqvist, U., Sato, K., et al. (2012) A SHORT INTERNODES (SHI) family transcription factor gene regulates awn elongation and pistil morphology in barley. J. Exp. Bot. 63: 5223-5232.

Zhang, D., Kong, W., Robertson, J., Goff, V.H., Epps, E., Kerr, A., et al. (2015) Genetic analysis of inflorescence and plant height components in sorghum (Panicoidae) and comparative genetics with rice (Oryzoidae). BMC Plant Biol. 15: 107.

Zhang, Y.P., Zhang, Z.Y., Sun, X.M., Zhu, X.Y., Li, B., Li, J.J., et al. (2019) Natural alleles of GLA for grain length and awn development were differently domesticated in rice subspecies japonica and indica. Plant Biotechnol. J. 17: 1547-1559.

Zhou, L., Zhu, C., Fang, X., Liu, H., Zhong, S., Li, Y., et al. (2021) Gene duplication drove the loss of awn in sorghum. Mol. Plant doi: 10.1016/j.molp.2021.07.005. 
bioRxiv preprint doi: https://doi.org/10.1101/2021.09.29.462495; this version posted October 2, 2021. The copyright holder for this preprint (which was not certified by peer review) is the author/funder. All rights reserved. No reuse allowed without permission.

Table 1. Summary of awn-related quantitative trait loci (QTLs) detected in this study.

\begin{tabular}{|c|c|c|c|c|c|c|c|c|c|}
\hline Trait & Year_location & Chr & QTL ID & $\begin{array}{l}\text { Position } \\
\text { (cM) }\end{array}$ & Nearest marker & Marker interval & LOD & $\begin{array}{l}\text { PVE } \\
(\%)\end{array}$ & $\mathbf{A E}$ \\
\hline \multirow{3}{*}{$\begin{array}{l}\text { Awn length } \\
\text { (AL) }\end{array}$} & 2014_OKY & 3 & $q A L 3$ & 76.62 & Chr03:72655415 & Chr03:71923025-72847396 & 116.4 & 90.6 & 5.75 \\
\hline & 2015_TKY & 3 & $q A L 3$ & 76.62 & Chr03:72655415 & Chr03:71923025-72847396 & 100.8 & 88.7 & 4.58 \\
\hline & 2014_OKY & 9 & $q A L 9$ & 59.76 & Chr09:57681027 & Chr09:56467317-58331590 & 7.0 & 1.3 & 0.64 \\
\hline \multirow{2}{*}{$\begin{array}{l}\text { Awn presence } \\
\text { (AP) }\end{array}$} & 2014_OKY & 3 & $q A P 3$ & 76.62 & Chr03:72655415 & Chr03:71923025-72847396 & 179.1 & 97.8 & 0.49 \\
\hline & 2015_TKY & 3 & $q A P 3$ & 76.62 & Chr03:72655415 & Chr03:71923025-72847396 & 120.8 & 92.7 & 0.48 \\
\hline \multirow{4}{*}{$\begin{array}{l}\text { Awn length } \\
\text { (awned lines only) }\end{array}$} & 2014_OKY & 7 & $q A L 7$ & 47.27 & Chr07:59733246 & Chr07:58230302-60603839 & 8.3 & 23.8 & 1.06 \\
\hline & 2015_TKY & 7 & $q A L 7$ & 47.40 & Chr07:59733246 & Chr07:58230302-60603839 & 3.5 & 10.7 & 0.64 \\
\hline & 2014_OKY & 9 & $q A L 9$ & 59.76 & Chr09:57681027 & Chr09:56467317-57917278 & 5.9 & 16.2 & 0.87 \\
\hline & 2015_TKY & 9 & $q A L 9$ & 60.06 & Chr09:57681027 & Chr09:56467317-58331590 & 4.8 & 15.0 & 0.76 \\
\hline
\end{tabular}

OKY, Okayama; TKY, Tokyo. Marker intervals were estimated based on confidence intervals (2.0-LOD). PVE: Phenotypic variance explained. For the additive effects (AE), positive values indicate that alleles from NOG increased the trait score. 


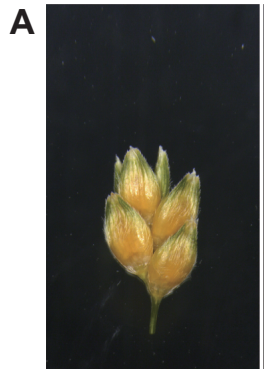

BTx623

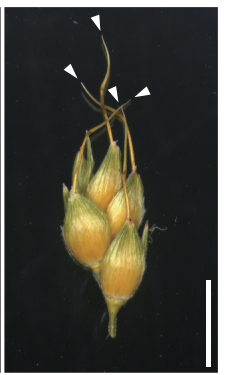

NOG
B

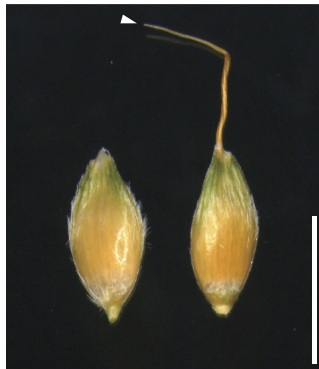

BTx623
C

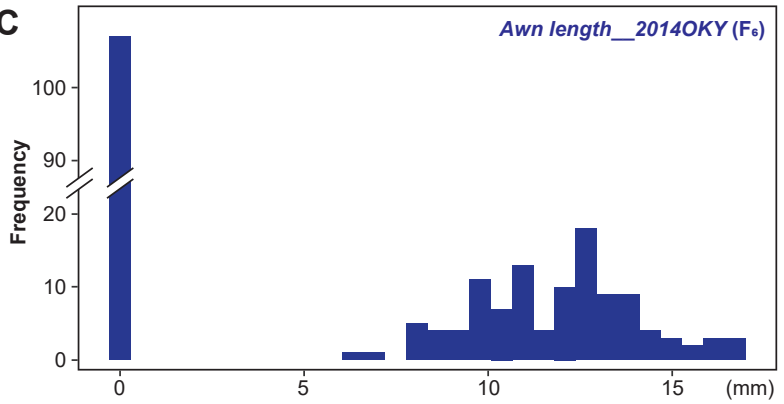

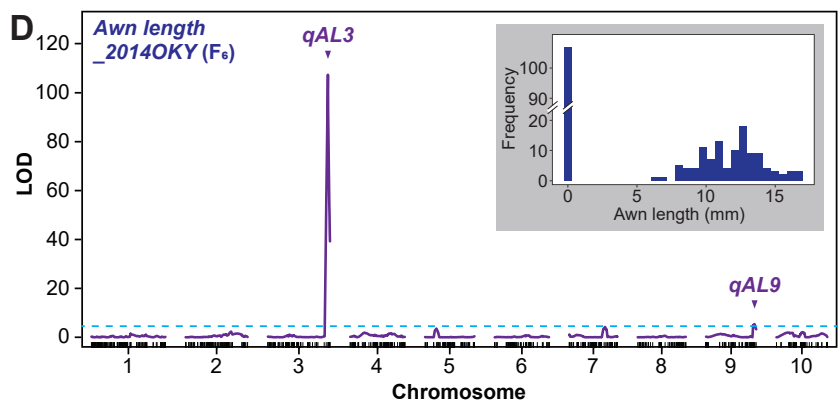
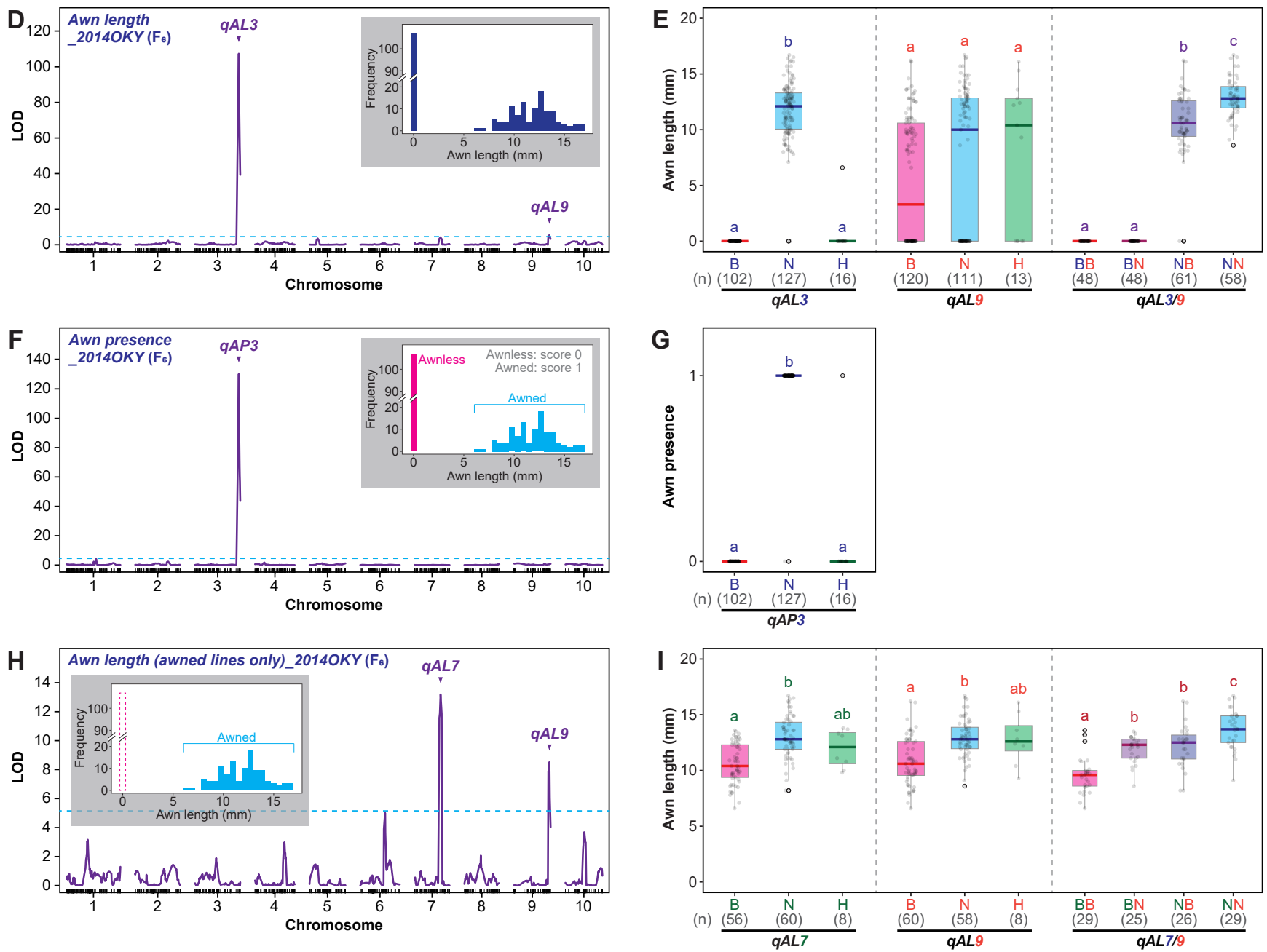

Figure 1

Quantitative trait locus (QTL) analysis for awn-related traits using the sorghum recombinant inbred line (RIL) population. (A) Secondary branches of BTx623 and NOG, parents of the RIL population. (B) Enlarged view of spikelets from BTx623 and NOG. Arrowheads indicate awn tips. (C) Frequency distribution of awn length (AL) in the RIL population ( $F_{6}$ generation) cultivated at Okayama in 2014. Results of QTL analysis for the awn-related traits $(D, F, H)$ and their allelic effects $(E, G, I)$. (D, E) AL of all lines, $(F, G)$ awn presence $(A P),(H, I) A L$ of awned lines only. (D, F, H) LOD profiles obtained from composite interval mapping (CIM). Horizontal dotted lines represent a threshold of the $1,000 \times$ permutation test $(P<0.05)$. (E, G, I) Contributions of SNP genotypes of the nearest marker for significant QTLs. Box plots show effects of the nearest marker genotypes for each QTL or allelic combinations of QTLs. B, BTx623-type homogeneous allele; N, NOG-type homogeneous allele; $\mathrm{H}$, heterogeneous. The number of lines that corresponded to each genotype is shown in parentheses. Different letters denote significant differences according to the Tukey-Kramer test $(P<0.01)$. Scale bars $=5 \mathrm{~mm}$ in $(A)$ and $(B)$. 
A $\quad \begin{gathered}\text { RIL205 }\left(F_{6}\right) \\ \text { awnless }\end{gathered}$

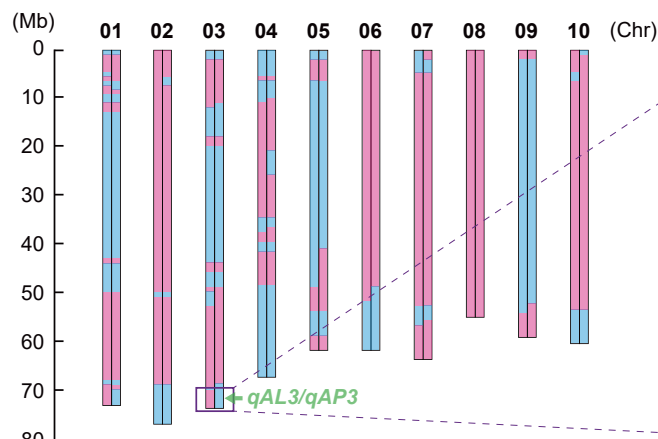

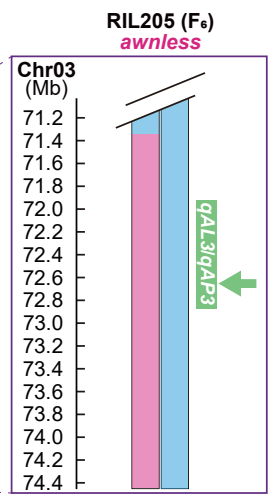

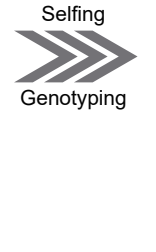

Stage 5
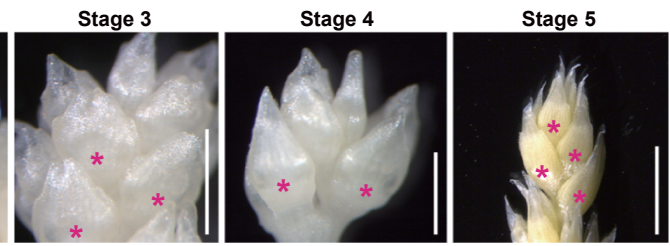

Stage 6

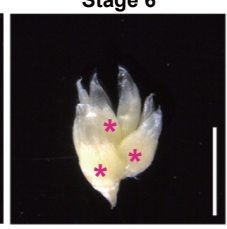

Stage7
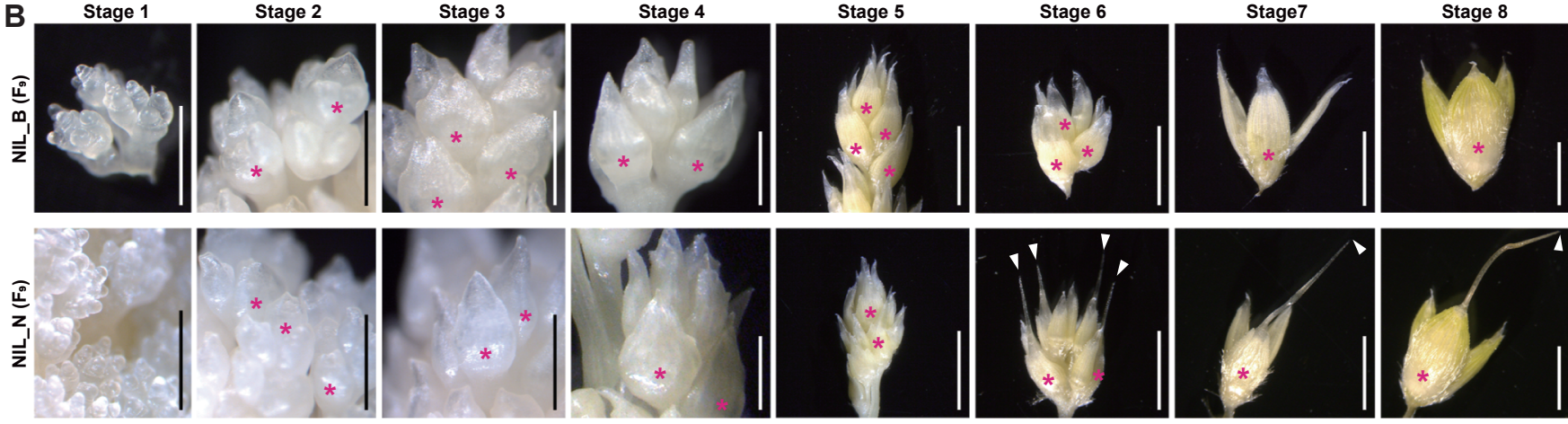

C
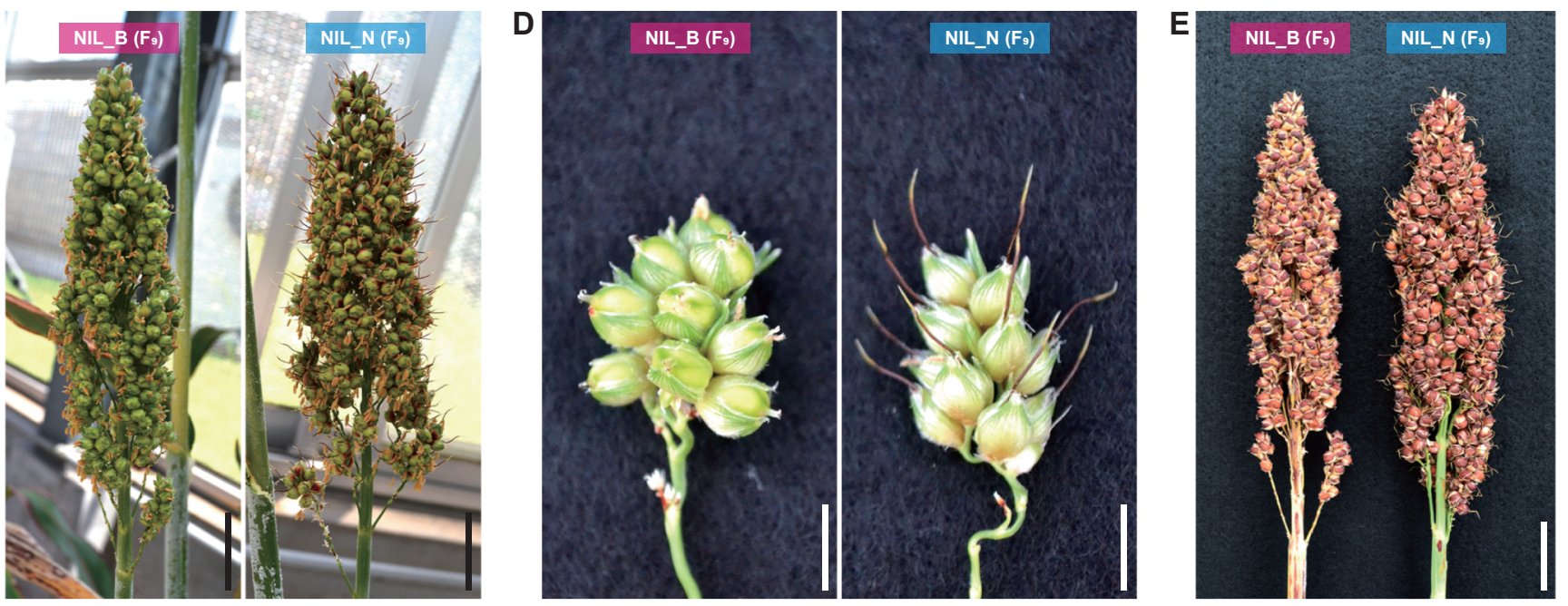

Figure 2

Reconfirming the effect of $q A L 3 / q A P 3$ using near isogenic lines (NILs). (A) Graphical genotypes of RIL205 (F6, awnless), one of the heterozygous lines at the genotype of the marker associated with qAL3/qAP3 (left), and magnified view of the Chr03 terminus for RIL205, NIL with the homogeneous BTx623 allele (NIL_B, F9, awnless), and that with the homogeneous NOG allele (NIL_N, F9, awned) at $q A L 3 / q A P 3$ (right). Magenta and cyan boxes indicate genomic regions from BTx623 and NOG, respectively. PCR genotyping using insertion-deletion polymorphisms revealed that recombination occurred between Chr03:72369727-Chr03:72556683 in both NILs; differences between NILs at the Chr03 terminus were approximately 1.83-2.02 Mb. "Common" indicates that the genotypes (homogeneous allele) are common between both NILs, although we could not determine whether they are BTx623-type or NOG-type by PCR genotyping. A bar and arrow in green indicate the marker interval and the position of the nearest marker of $q A L 3 / q A P 3$ in the QTL analysis. (B-E) Observations of spikelets and panicles of NILs at various growth stages. (B) Spikelets of young panicles. Staging of young spikelets was carried out based on the length of sessile spikelets (SSs, asterisks). Stage 1, primordial stage; Stage 2, 0.3-0.4 mm; Stage 3, 0.4-0.6 mm; Stage 4, 0.6-0.8 mm; Stage 5, 1.5-2.0 mm; Stage 6, 2.0-2.5 mm; Stage 7, 2.6-3.3 mm; and Stage 8, 3.7-4.2 mm, respectively. Arrowheads indicate awn tips. Scale bars = $0.5 \mathrm{~mm}$ in Stage 1-4, $2.0 \mathrm{~mm}$ in Stage 5-8. (C) Panicles at the grain-filling period. (D) Enlarged images of the primary branches at the grain-filling period. (E) Panicles at the mature stage. Scale bars $=3 \mathrm{~cm}$ in (C) and (E), $5 \mathrm{~mm}$ in (D). 


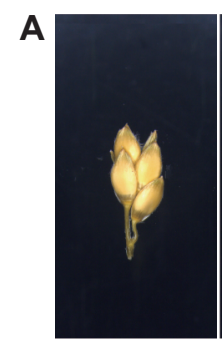

PI 152771

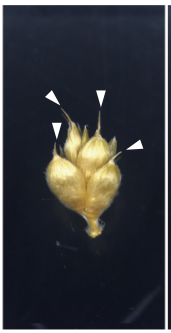

IS 11026

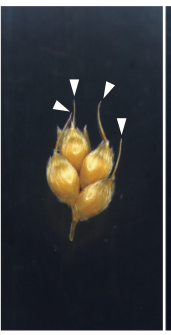

IS 27805

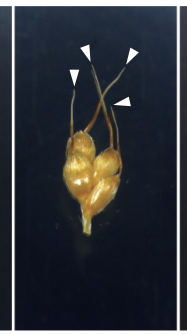

IS 22799

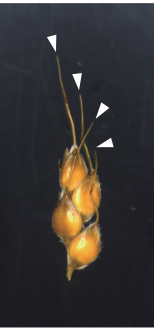

WSC 67

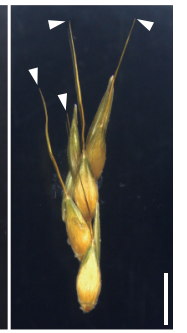

PI 180349
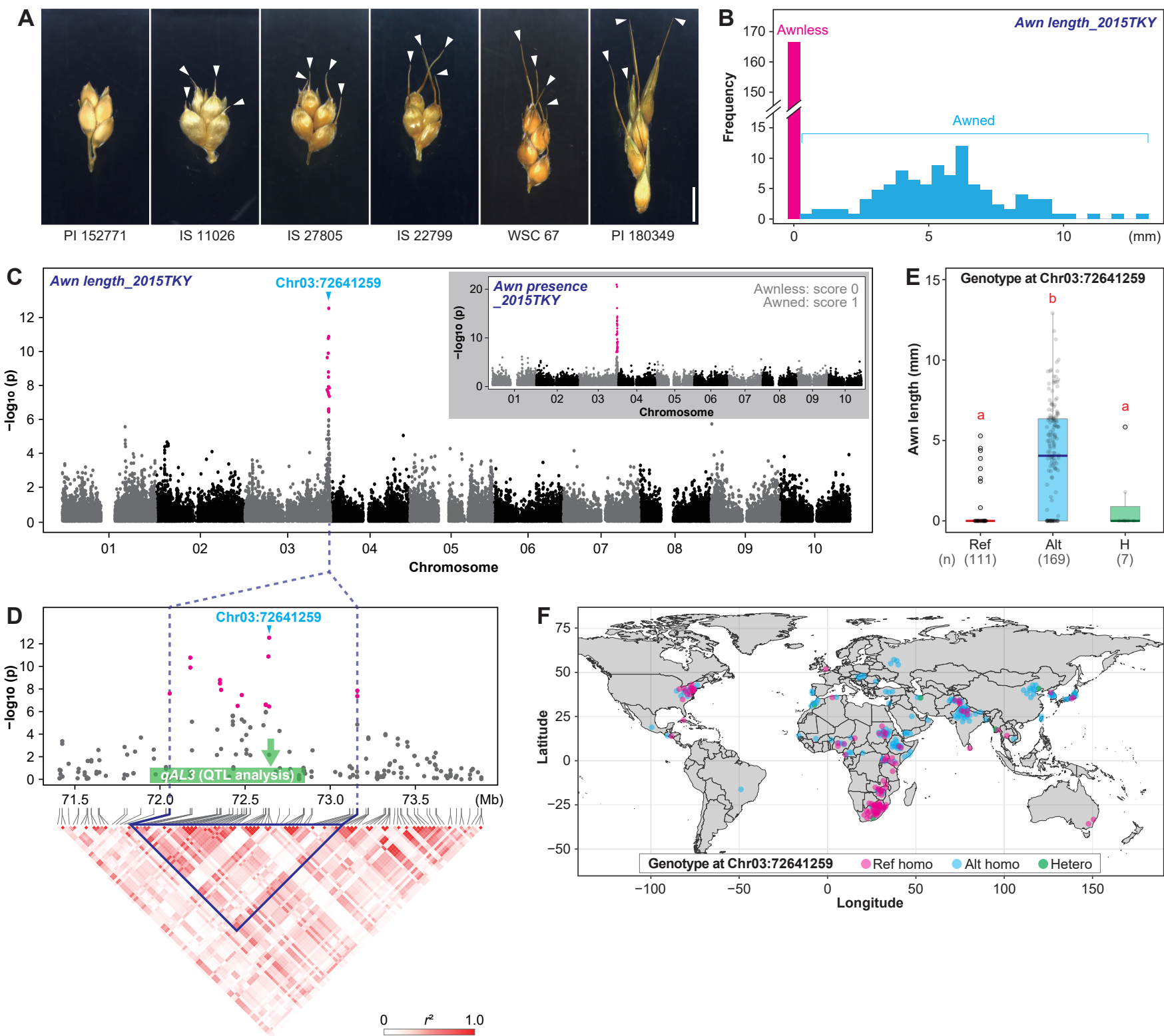

Figure 3

Genome-wide association study (GWAS) for awn-related traits using the sorghum germplasm. (A) Examples of secondary branches from the panicles of sorghum germplasm. Accession ID is shown on the bottom of each panel. Arrowheads indicate awn tips. (B) Frequency distribution of AL in the sorghum germplasm cultivated at Tokyo in 2015. (C) Manhattan plot for AL and AP (insert). Dots in magenta indicate the single-nucleotide polymorphisms (SNPs) that are significantly associated with traits (Benjamini-Hochberg-corrected significance; $P<$ 0.001). (D) Local Manhattan plot (top) and LD heatmap (bottom) surrounding the peak on chromosome 3. A bar and arrow in green indicate the marker interval and the position of the nearest marker of $q A L 3 / q A P 3$ in the QTL analysis. (E) Box plot shows the effects of the peak SNP (Chr03:72641259) genotype for AL. Ref, reference-type homogeneous allele; Alt, altered-type homogeneous allele; $\mathrm{H}$, heterogeneous. The number of lines that corresponded to each genotype is shown in parentheses. Different letters denote significant differences according to the Tukey-Kramer test $(P<0.01)$. (F) Geographic distribution of alleles at the peak SNP (Chr03:72641259), color-coded by allele (Ref homo, magenta; Alt homo, cyan; Hetero, green). Scale bar $=5 \mathrm{~mm}$ in (A). 
A

NOG Peak of GWAS (Chr03:72641259)
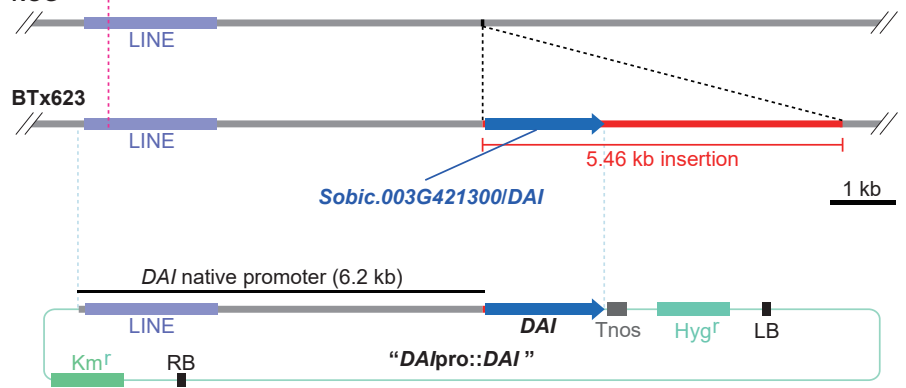

B

Sobic.003G421300IDAI

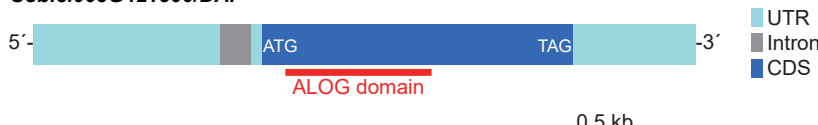

$0.5 \mathrm{~kb}$
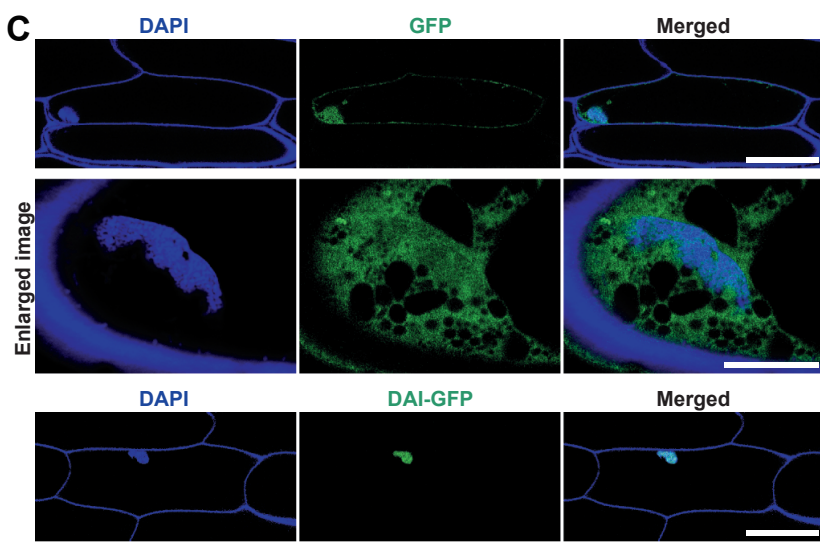

DAI-GFP

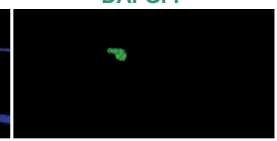

Merged
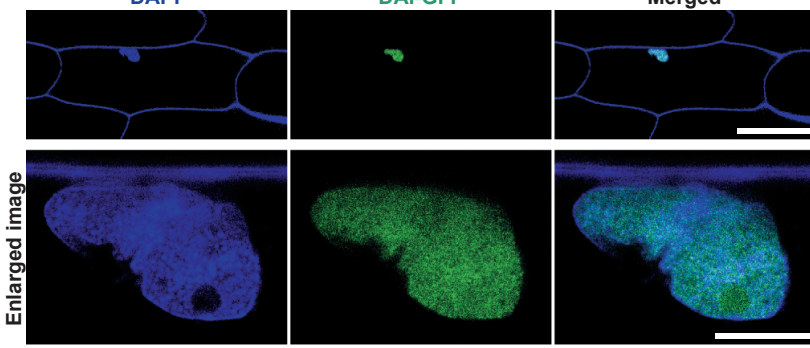

D

\begin{tabular}{|c|c|c|c|c|c|}
\hline Accession ID & Origin & Genotype at Chr03:72641259 & Phenotype & $\begin{array}{l}\text { Presence of } D A I \\
\text { (5.46 kb insertion) }\end{array}$ & Mutation in the CDS of $D A I$ \\
\hline PI 576320 & United States of America & A (Ref homo) & Awnless & Present & No mutation \\
\hline PI 533900 & Japan & A (Ref homo) & Awnless & Present & No mutation \\
\hline PI 295123 & Australia & A (Ref homo) & Awnless & Present & No mutation \\
\hline WSC 01 & Japan & A (Ref homo) & Awnless & Present & No mutation \\
\hline WSC 105 & Zimbabwe & A (Ref homo) & Awnless & Present & No mutation \\
\hline PI 180349 & India & G (Alt homo) & Awned & Absent & - \\
\hline PI 173120 & Turkey & G (Alt homo) & Awned & Absent & - \\
\hline PI 586554 & China & G (Alt homo) & Awned & Absent & - \\
\hline PI 179052 & Turkey & G (Alt homo) & Awned & Absent & - \\
\hline PI 196597 & Taiwan & G (Alt homo) & Awned & Absent & - \\
\hline PI 656029 & United States of America & A (Ref homo) & Awned & Absent & - \\
\hline IS 24453 & South Africa & A (Ref homo) & Awned & Present & C to $T$ substitution at $82^{\text {nd }}$ position ( $\mathbf{Q}_{28} \rightarrow$ Stop) \\
\hline PI 152771 & Sudan & G (Alt homo) & Awnless & Present & No mutation \\
\hline IS 29606 & South Africa & G (Alt homo) & Awnless & Present & No mutation \\
\hline
\end{tabular}

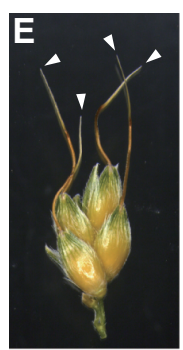

WT (RIL232)

$(-/-)$

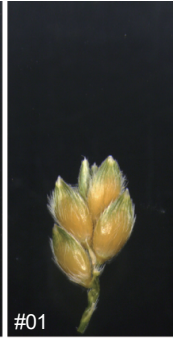

Homozygous (DAI/DAI)

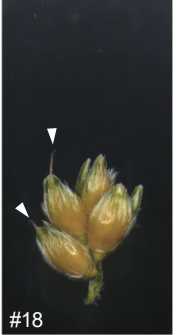

Heterozygous (DAl/-)

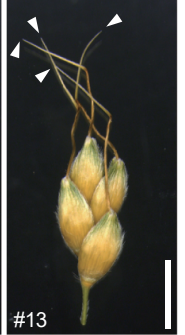

Azygous

$(-/-)$

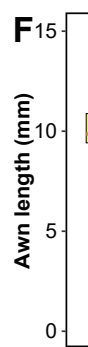

$(-/-)$

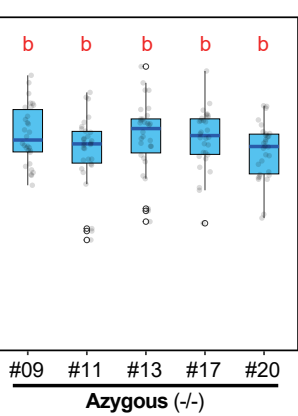

Figure 4

Identification of the Dominant Awn Inhibitor (DAI) gene. (A) Genomic structures surrounding DAl on chromosome 3 in the NOG and BTx623 genome (upper), and schematics of the $D A /$ pro::DAl plasmid for making transgenic sorghum (bottom). (B) The gene structure of $D A I$ in BTx623 is shown. Pale blue, gray, and blue boxes show UTR, intron, and CDS, respectively. A red line indicates a region coding the ALOG domain. (C) Localization of green florescent protein (GFP) (upper) and DAI-GFP fusion protein (bottom) in onion epidermal cells. (D) Table showing the relationship among genotype of the peak SNP in GWAS (Chr03:72641259), awn phenotype, presence of DAl, and mutations within the CDS of $D A l$ in 14 accessions of sorghum germplasm. (E) Examples of secondary branches from panicles of the awned parental line (RIL232), homozygous transgenic plant (DAI/DAI), heterozygous transgenic plant (DAI/-), and azygous plant (-/-). Arrowheads indicate awn tips. (F) Box plot shows the AL of the RIL232, homozygous, heterozygous, and azygous plants. Different letters denote significant differences according to the Tukey-Kramer test $(P<0.01)$. Scale bars $=100 \mu \mathrm{m}$ in $(C ; 10 \mu \mathrm{m}$ in Enlarged images), $5 \mathrm{~mm}$ in $(E)$. 

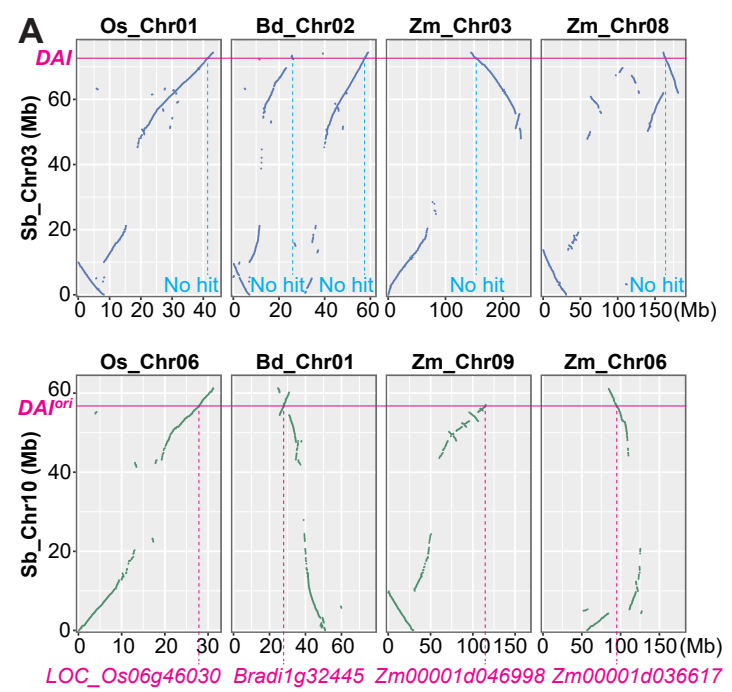

\section{B Sb_Chr03 $72.62-72.68 \mathrm{Mb}(\mathrm{f})$ \\ Os Chr01 \\ 41.30-41.40 Mb (f) \\ Bd_Chr02 \\ 25.90-25.97 Mb (r) \\ Bd_Chr02 \\ 57.56-57.60 Mb (r) \\ $\mathrm{Zm} \mathrm{Chr03}$ \\ 153.35-153.60 Mb (r) \\ Zm_Chr08 \\ 163.95-164.05 Mb (r)}

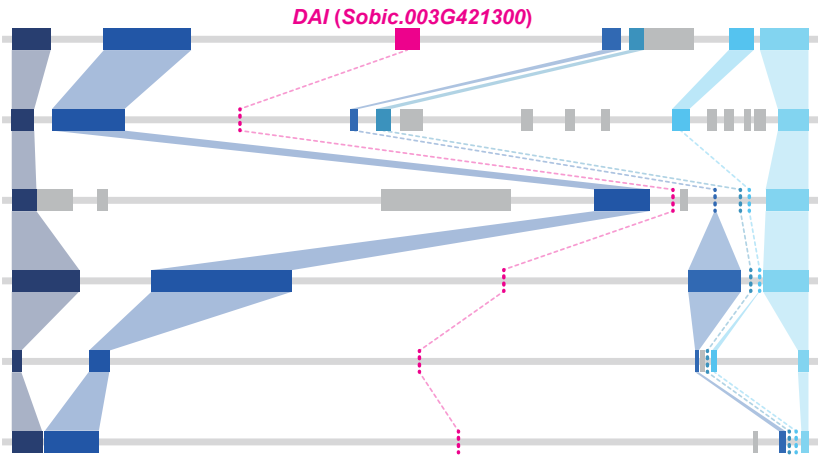

C
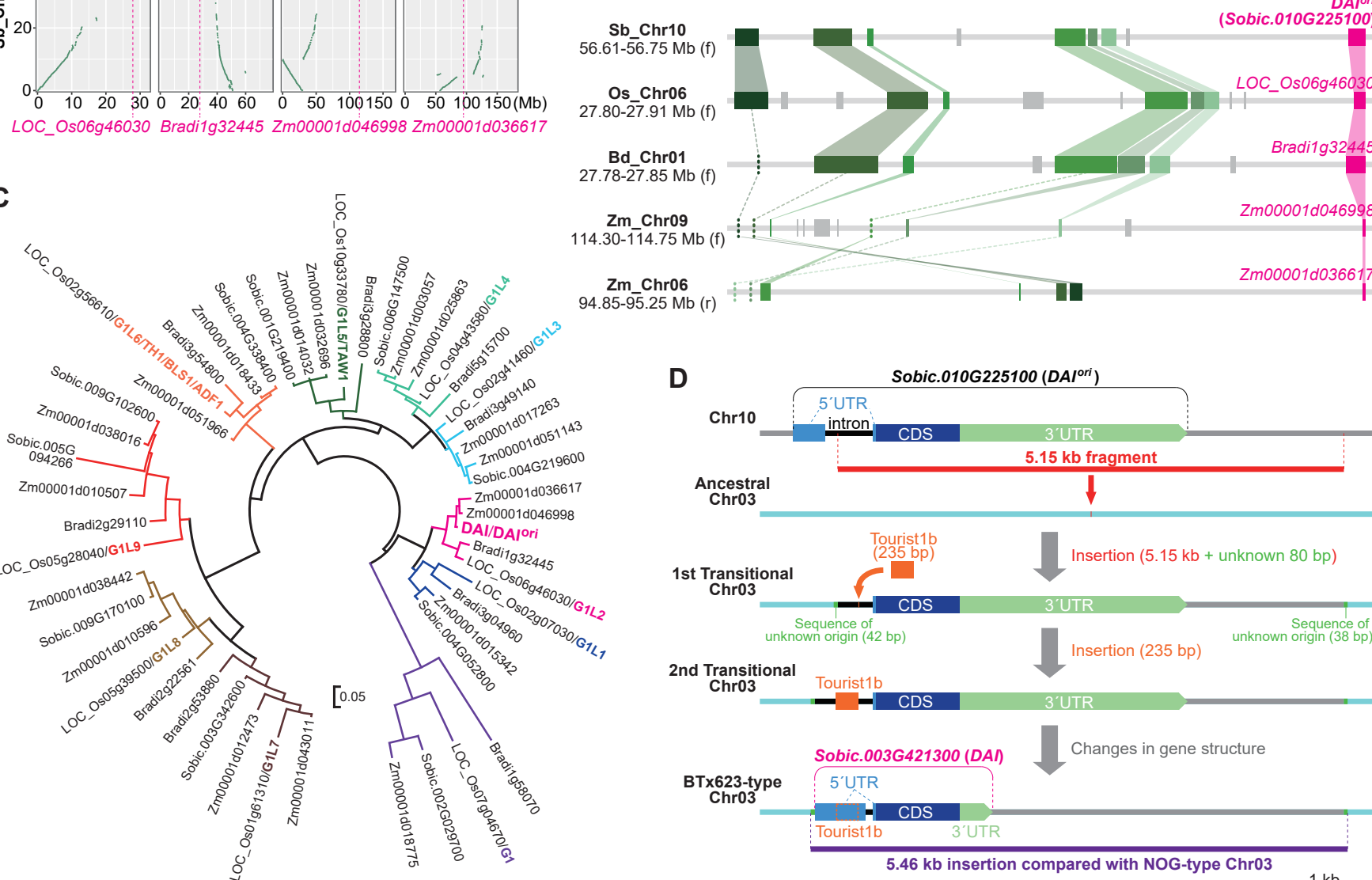

\section{Figure 5}

The birth and evolution of the $D A /$ gene in sorghum. $(\mathrm{A}, \mathrm{B})$ Synteny analysis for the $D A /$ gene and its paralogous gene, $D A /$ ori among four grass species. (A) Chromosome-level synteny analysis for sorghum chromosome 3 encoding $D A l$, and 10 encoding $D A /$ ori. Sb, Sorghum bicolor; Os, Oryza sativa; Bd, Brachypodium distachyon; and Zm, Zea mays. (B) Schematic diagram of the genomic structures around $S b D A I, S b D A l^{\circ r i}$, and their syntenic regions in four grass species. Boxes in the same color indicate that they are orthologous genes. Genes shown in dotted lines indicate that corresponding genes do not exist in this region. ' $f$ ' and ' $r$ ' indicate forward and reverse strand, respectively. (C) Phylogenetic tree for DAI/DAlori and related proteins from the four grass species. (D) A model for the birth and evolution of the DAl gene. Gene structures of $D A I$ and $D A /$ ori are based on the information of the reference genome of Sorghum bicolor (v3.1.1). 
A NOG

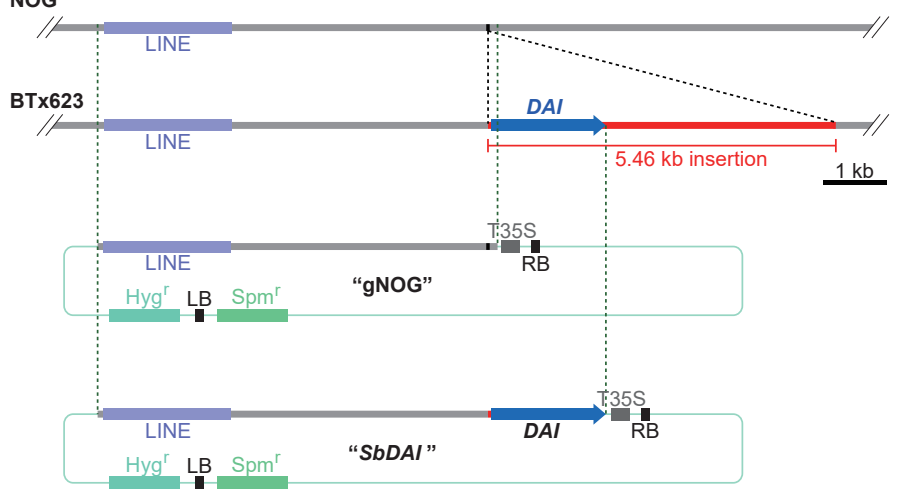

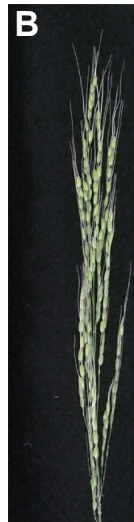

WT (Kasalath)
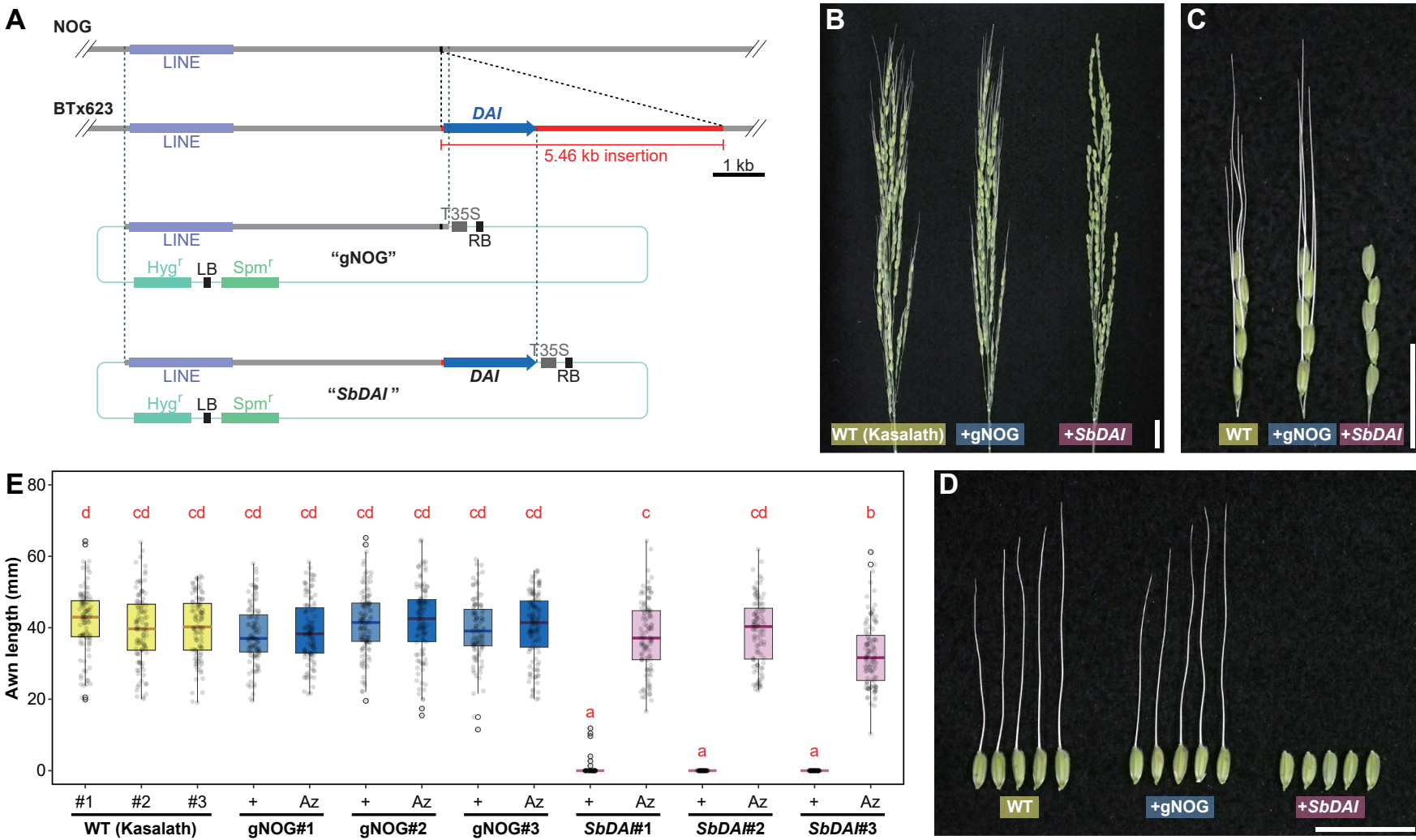

Figure 6

$\mathrm{SbDAl}$ can also inhibit awn elongation in Kasalath. (A) Schematics of the gNOG and $\mathrm{SbDA}$ / plasmids for making transgenic Kasalath. (B-D) Examples of panicles (B), secondary branches (C), and spikelets (D) of the wild-type Kasalath, the gNOG transgenic line, and the SbDAI transgenic line. (E) Box plot shows the AL of the wild-type Kasalath, gNOG transgenic lines, $S b D A /$ transgenic lines, and their azygous lines. ' + ' or 'Az' indicate the individual has the transgene or does not, respectively. Different letters denote significant differences according to the Tukey-Kramer test $(P<0.01)$. Scale bars $=2 \mathrm{~cm}$ in $(B-D)$. 
bioRxiv preprint doi: https://doi.org/10.1101/2021.09.29.462495; this version posted October 2, 2021. The copyright holder for this preprint

A
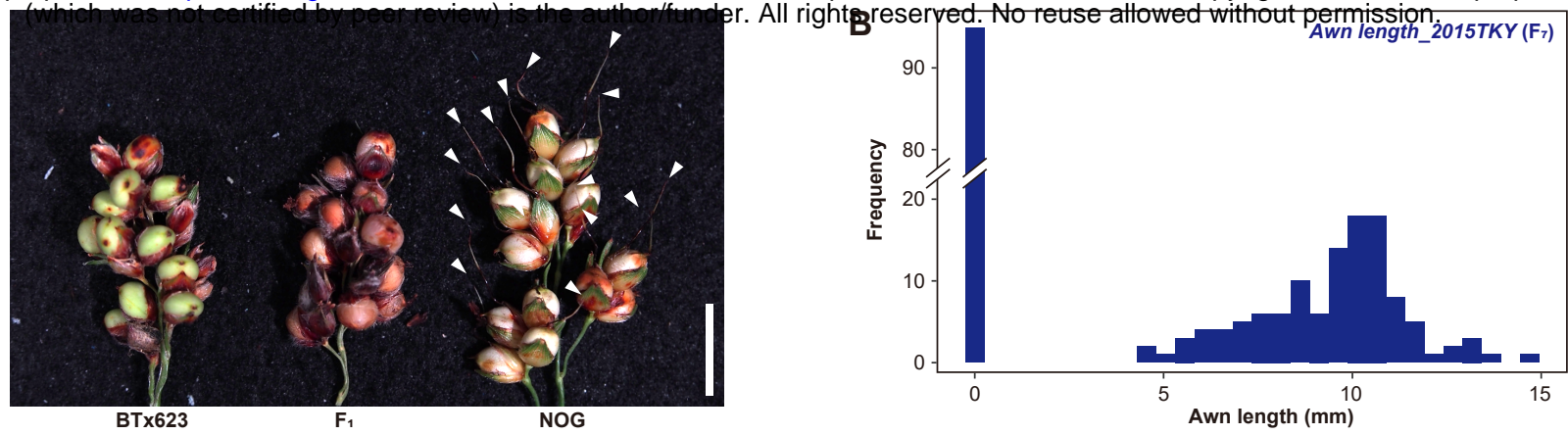

C
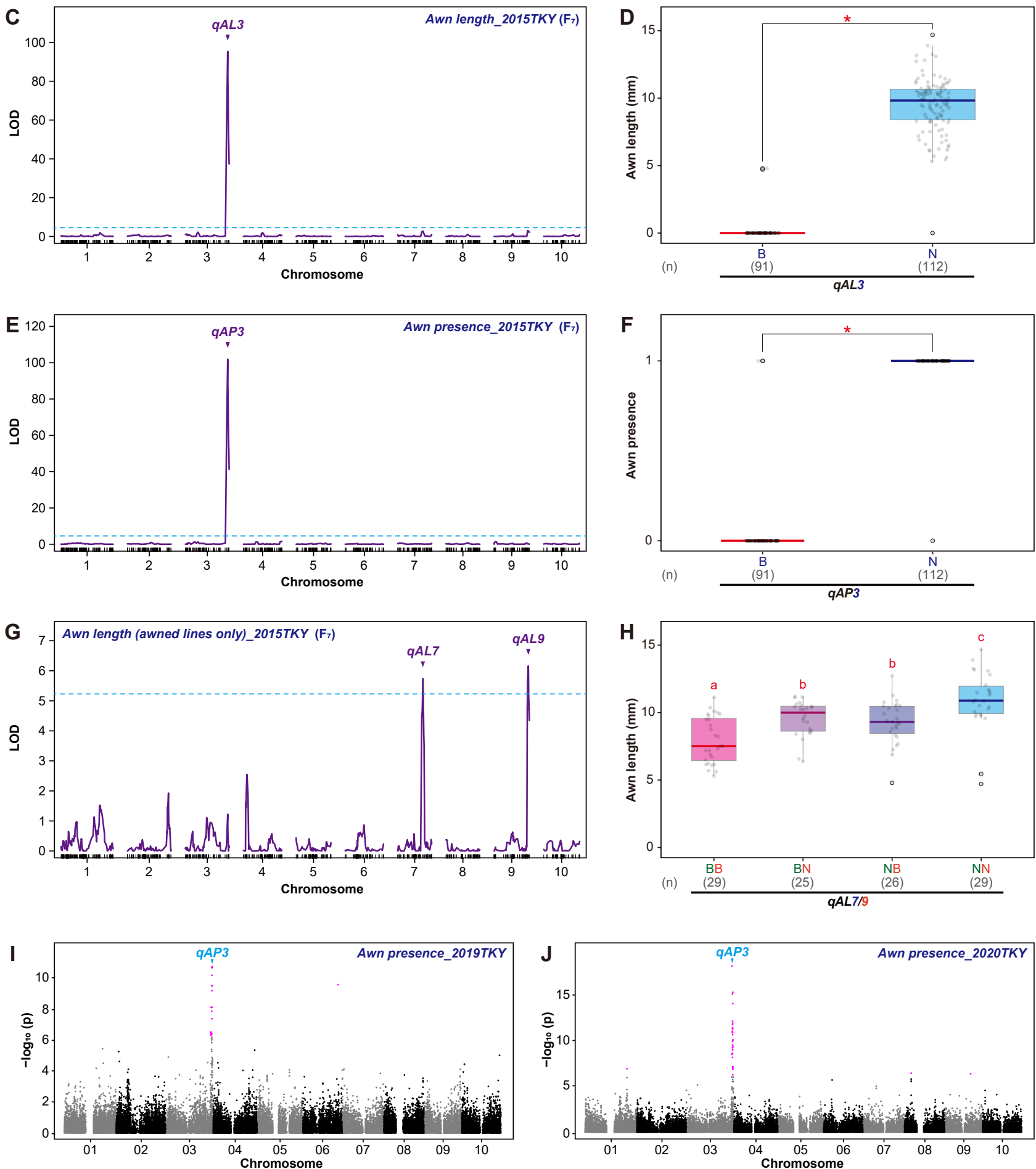

\section{Supplementary Figure 1}

Results of reproducibility check of QTL analysis and GWAS using lines cultivated in different years [2015 at Tokyo for RILs ( $F_{7}$ generation), 2019 and 2020 at Tokyo for germplasm] from those in Fig. 1 and 2. (A) Awn phenotype of $F_{1}$ and its parents. Arrowheads indicate awn tips. (B) Frequency distribution of awn length in the RIL population ( $F_{7}$ generation) cultivated at Tokyo in 2015. Results of QTL analysis for the awn-related traits (C, E, G) and their allelic effects (D, F, H). (C, D) Awn length of all lines, $(E, F)$ awn presence, $(G, H)$ awn length of awned lines only. (C, E, G) LOD profiles obtained from composite interval mapping (CIM). Horizontal dotted lines represent a threshold of the $1,000 \times$ permutation test $(P<0.05)$. (D, F, H) Contributions of SNP genotypes of the nearest marker for significant QTLs. Box plots show the effects of the nearest marker genotypes for each QTL or allelic combinations of QTLs. B,

BTx623-type homogeneous allele; N, NOG-type homogeneous allele. The number of lines that corresponded to each genotype is shown in parentheses. Asterisks indicate significant differences between genotypes (Welch's $t$-test, $P<0.001$ ). Different letters denote significant differences according to the Tukey-Kramer test $(P<0.05)$. (I, J) Manhattan plots for awn presence in 2019 and 2020. Dots in magenta indicate the SNPs that were significantly associated with traits (Bonferroni-corrected significance, $P<0.001)$. Scale bar $=1 \mathrm{~cm}$ in $(A)$. 
A bioRxiv preprint doi: https://doi.org/10.1101/2021.09.29.462495; this version posted October 2, 2021. The copyright holder for this preprint

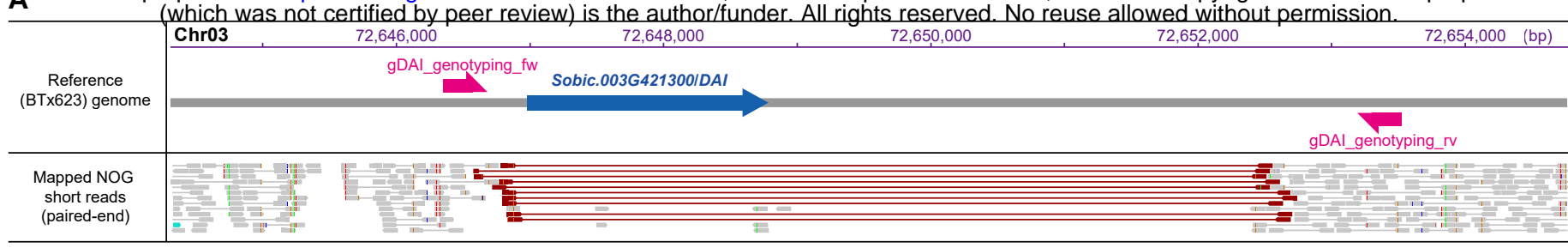

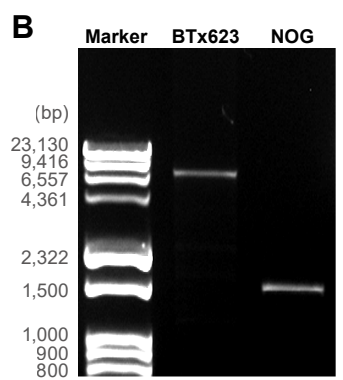

D

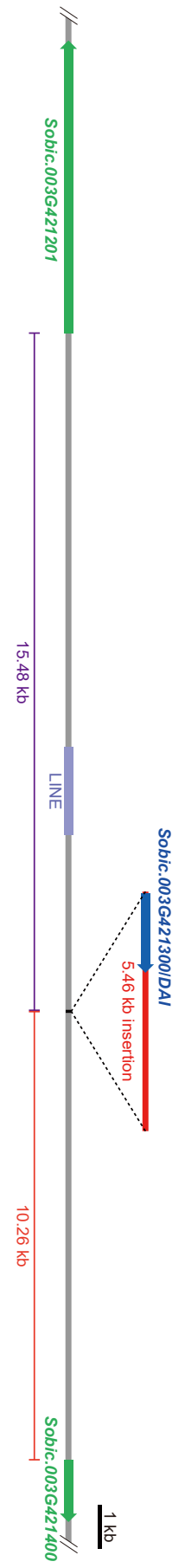

C

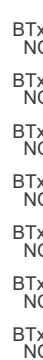
NOOG BTEG BTE23 Conserved ed region Inserted region in the BTx623 genome

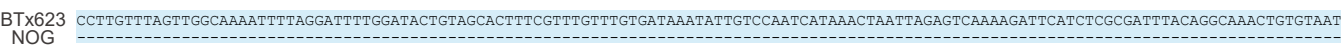

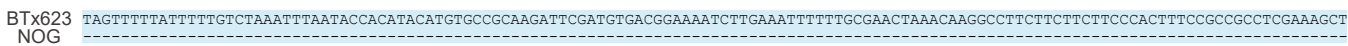

BTX623 TCTCTCCCTTCTCTTCTGCACGAGCATGTTGGTGATGGCGGCGAAAAGCTCAGTTTTTTTTTTGACTCCCCGGCGTTGTCGTCGCACTAATCGAGGGGGGTTGCATGCAGATCTGAGGCGCGAGGAGCAAGCG
NOG -

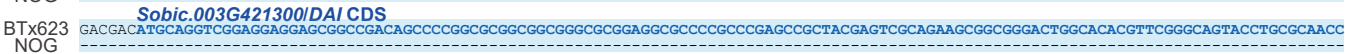

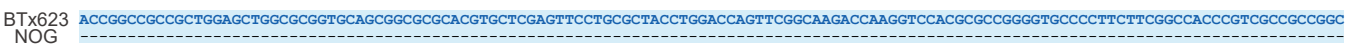
BTX623 BTX623 CTCCGCGAGGTCCGCGACAGCCAGGCCAAGGCGCGCGGCATCGCCTACGAGAAGAAGCGCCGGAAGCGGCCGTCCGCTTCGTCGTCCCAGTCGTCGCCGCAGGCCGCGACGACCCCGCCGCAGCAGGCTCCTC NOG BTX623 CCGTCTCCTCGCCGGCCCTGTCGGACGTGGTGGCCGAGAGGGCCGACGTGCGGGCGCACGTGCCGGACGCCGGGCATCAGCAGCACCATCACCTGCACCAGCACCAGCACCAGCATCACTTCTTCATGCCACA
NOG BTX623 CCCGCAGTTCCTGCACGGGTTCAGCCTGCTGCCGGGCAACCCCGAAGCGGTGGCTGCCAATGGCAACGGCGGCGGCAGCAGCAGCGCTAGCGTCGCTGCCGGCAACGGTGACGAGATAGCGCTGGCGATGGCG BTX623 GCGGCGGCGGAGGCGCACGCGGCGGGGTGCATGCTGCCGCTGTCGGTGTTCAACTAGGCAGGCAAGCAGGCGTATAGCCAGGCGAGGTAGGGCTTAAACAATAGTAGATTAATCCGGGTGCTTATCTAGTGTT

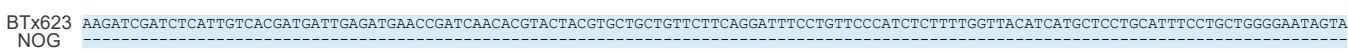
BTX623 TTAGTAGCTGTGCTTGGTACTGTAGGGTTTGCAGAATCCTGCTTGGTTTGTGTATGCAATGCAATGATGGTGGAGATATAATATAATGGAAGGCTTAATTTCCAGACTAATGTTCTTGATGCATTCTTTCTCT
NOG BTX623 CTGTGATTAATTAATTTATTGGATATCTGATTCGTACTAAGGGCCTCTTTGGTTCCCCTTACTAAGTTTTAGCTAGCTAAAATTATTTTAGCTACTCTTGGGTGACTAATGGGACTAAATATTTTAGCTCCTT
NOG

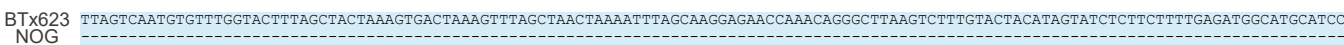
BTX623 TTTTAATTTTTGTCCCAGTATATATGCACACTCTTGGACGATCAAGTGGTTGCTCGATTTTTCAGGTATGCATTGCATTCAGCTGAGAGAGCCTCGTACAAATCTTGACTAAAGTTAGTTGTGGTAGATTATA
NOG

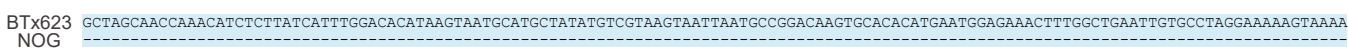

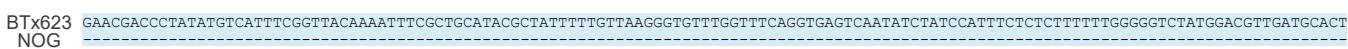

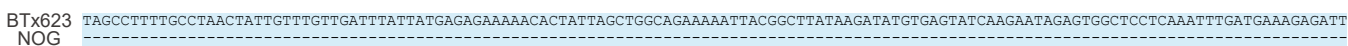

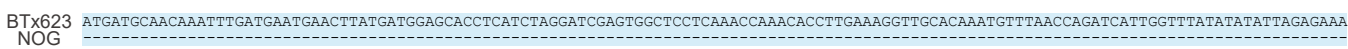

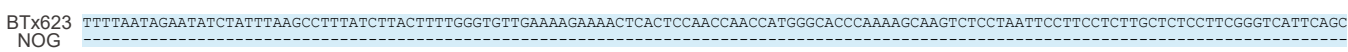
BTX623 TCATGCGCCGTCTCCGTCGCTACCCGCCTCAAACTCGACCTATCCCACTTGCCTTGTCAAATTAAGAATGCTAAGCAGAACCCTAAATCTGGTAGCTAGAGATCGGCTTCCCTGCACGCCATCCTTGTGCCGA
NOG BTX623 TCACCAAGAGGTCATCCCCTGTTGCACACCATGGTGAGTTGCAATTAGCTCGAGCTTAGCCTTCCCTGCTGCCATCTTCCCTTCGGCATCCAGGCTCTTTCTCGGATAGCCCTTTATCCAATCTCAGCCTCCC
NOG

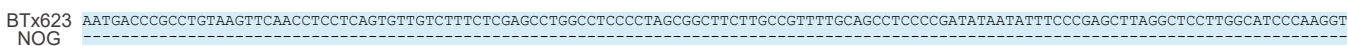

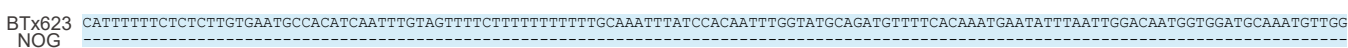
BTX623 ATGCAAGAGGATTCGATGATGACGATGATGATGATAATAACTTAGCTTCATTCCAGCAACTGCTTTGGTTGCCGAAGTTGAATAGAGAAGGCAAAGTAGGGGTGCCGACCTTGAATGAAGATGACATGTTTGA

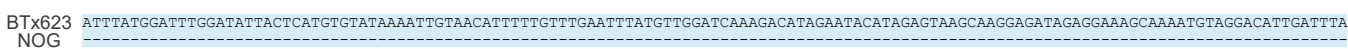

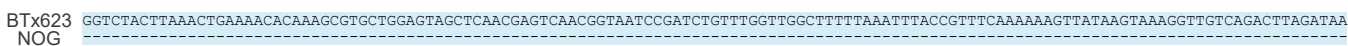

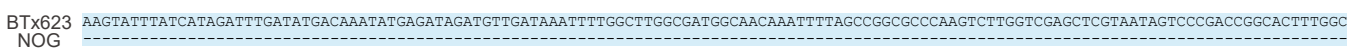

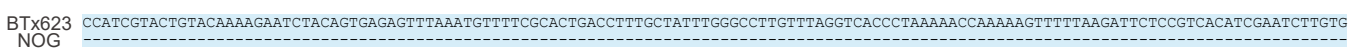

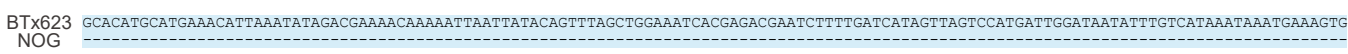
BTX623 CTACAGTACCGAAAACTTTTCACTTTTCGGAACTAAATAAGGAGAAGTTGTTGCCACAGTATGGGTGAATAAAAGCCTTCAAACAAGAATGACTAGGGTAGCTTTATTGGCGCTGTGCATGGCTCCACTTGGG
NOG BTX623 CGAAAACAGGCAGCATCATCATTGACTTAATTGGTGTGCCACTGTGCGTACAGTGAGAGGTTTTTGCAACAGAGCTCCGTATTTAGTGTGCATTCGCGACAAAAGGGGATTAAGGAGGAAATCTTAATCACCA BTX623 AGTGTCATGATTTACTGTTCAACCCAAGAAGAGCGTACGCCGGCCATCTCTCATTGTTTGTGCACTGTGTACTGATTGATTCCGCTAGAAAACAGTAACCAAGTCCGAAATAGGAAAAGACGGGGAAACCTTG

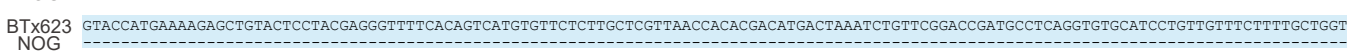
BTX623 TGCGCCAAATCGTGACAACTTGTTTATAATAGTTTCCAAACCTAGCTAGGCACACACTCCACTGGTCATGTCTTACTCCTATATATACCTTCAAAAACGTTGGTCCTTCTAATTTTATCCTAAATTAAGATTT
NOG

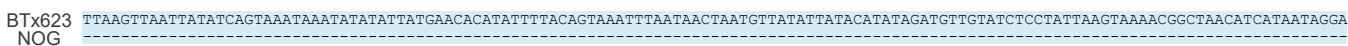

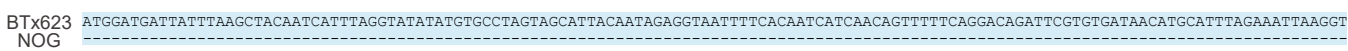

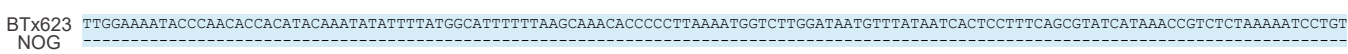

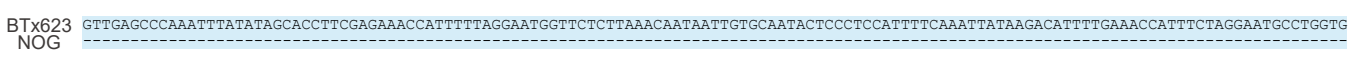

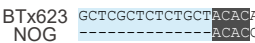

\section{Supplementary Figure 2}

Difference between genomic structures of BTx623 and NOG around the DAI gene. (A) Re-sequencing data of NOG in the region around DAI visualized using Integrative Genomics Viewer. Pentagonal boxes represent each short read, and the paired reads are connected to each other by lines. The paired reads shown in red indicate that the seize of inserts are unexpectedly large, i.e., NOG genome does not have regions between them. (B) Result of genotyping of BTx623 and NOG by PCR using primers indicated in (A). (C) Details of the genomic structure of NOG and BTx623 around the DAl gene as revealed by Sanger sequencing of PCR products in (B). (D) Insertion position of the 5.46 kb fragment and its relationship to nearby genes. 
bioRxiv preprint doi: https://doi.org/10.1101/2021.09.29.462495; this version posted October 2, 2021. The copyright holder for this preprint (which was not certified by peer review) is the author/funder. All rights reserved. No reuse allowed without permission.

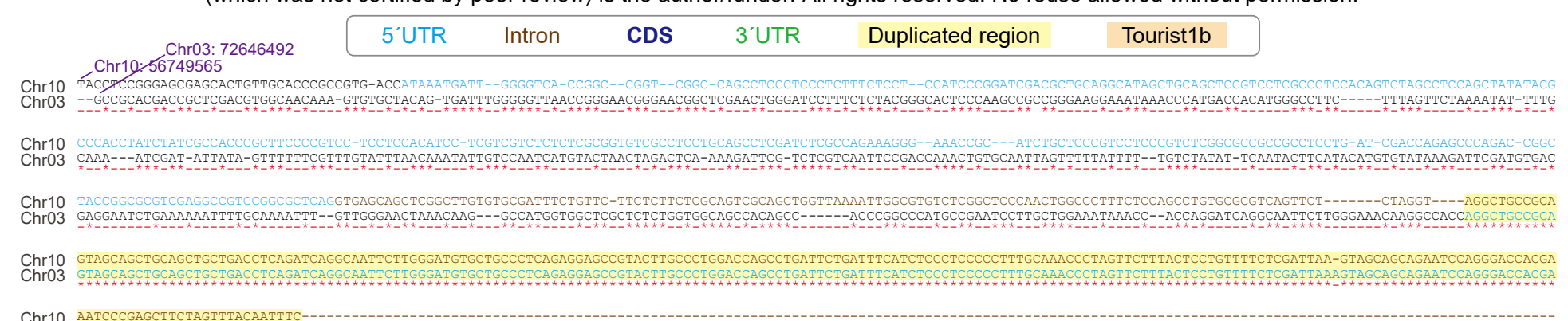

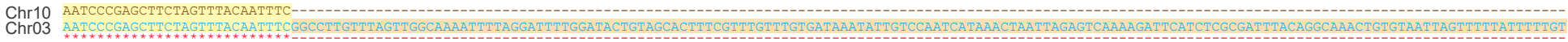

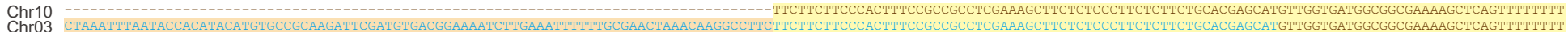

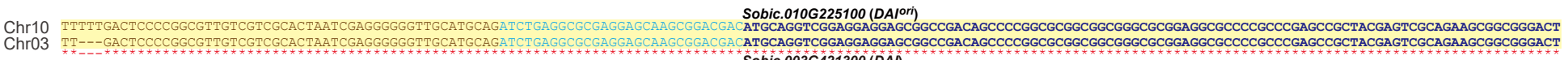

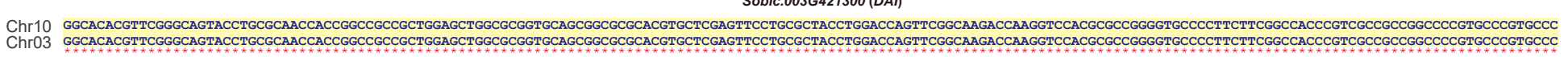

Chr10 GCTGCGCCAGGCCTGGGGCAGCCTCGACGCGCTCGTCGGCCGCCTGCGCGCCGCCTTCGAGGAGCACGGGGGACGGCCTGAGGCCAACCCCTTCGGCGCGCGCGCCGTGCGCCTATACCTCCGCGAGGTCCGCGACAGCCAGGCCAAGGCGCGCGGCATCGCCTACGAGAAGAAGCGC

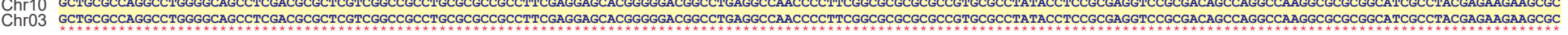

Chr10 CGGAAGCGGCCGTCCGCTTCGTCGTrCCAGTCGTCGCCGCAGGCCGCGACGACCCCGCCGCAGCAGGCTCCTCCCGTCTCCTCGCCGGCCCTGTCGGACGTGGTGGCCGAGAGGGCCGACGTGCGGGCGCACGTGCCGGACGCCGGGCACCAGCAGCACCATCACCTGCACCAGCACC

Chr10 AGCACCAGCATCACTTCTTCATGCCACACCCGCAGTTCCTGCACGGGTTCAGCCTGCTGCCGGGCAACCCCGAAGCGGTGGCTGCCAATGGCAACGGCGGCGGCAGCAGCAGCGCTAGCGTCGCTGCCGGCAACGGTGACGAGATAGGGCTGGCGATGGCGGCGGCGGCGGAGGCGCA

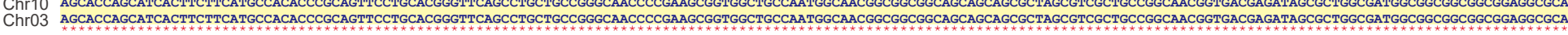

Chr10 CGGGGGGGGGTGCATGCTGCCGCTGTCGGTGTTCAACTAG

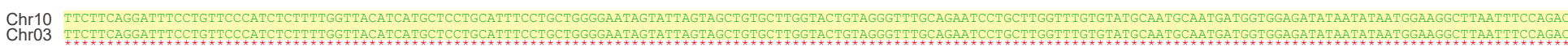

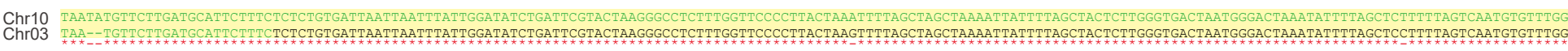

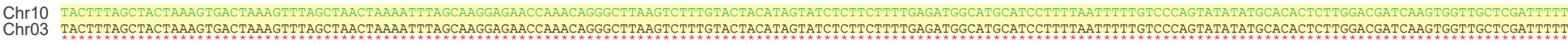

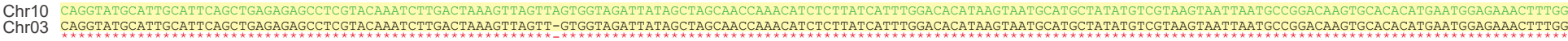

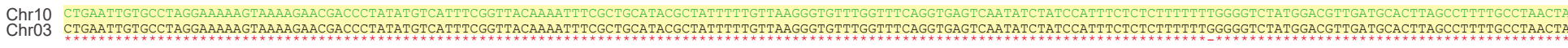

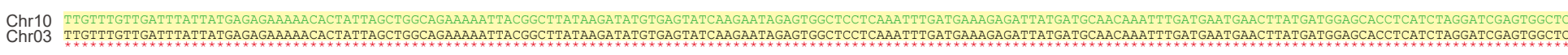

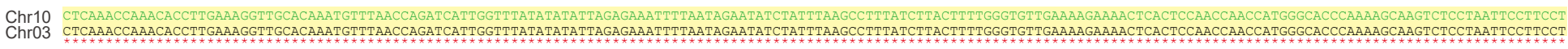

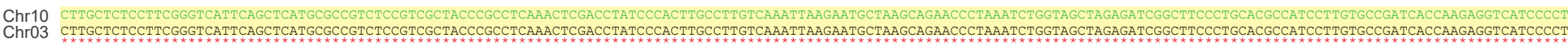

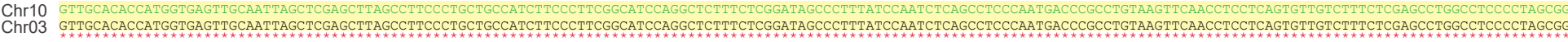

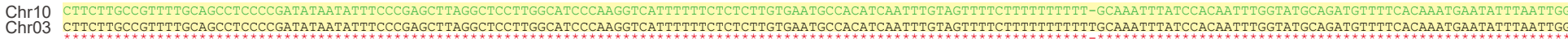

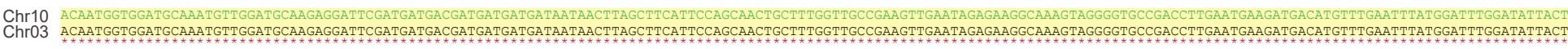

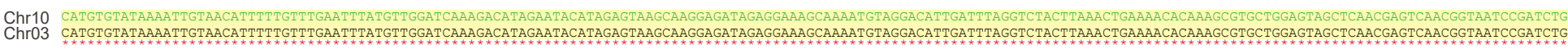

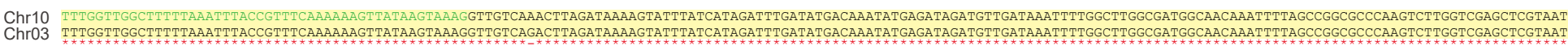

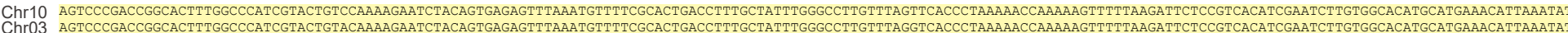

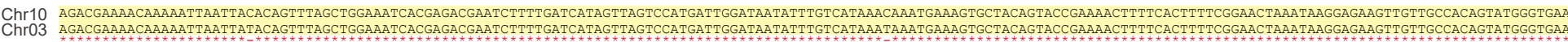

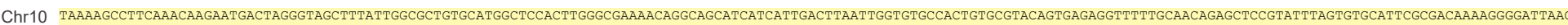

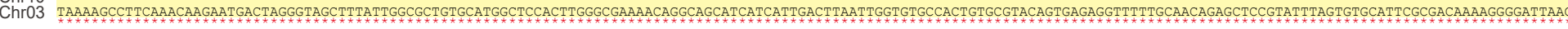

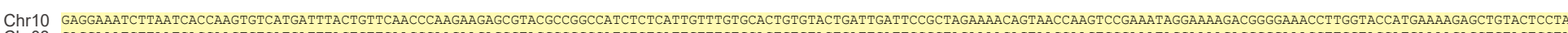

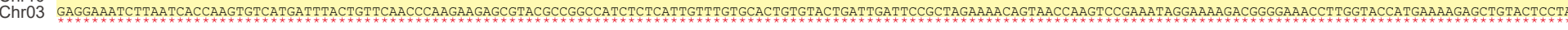

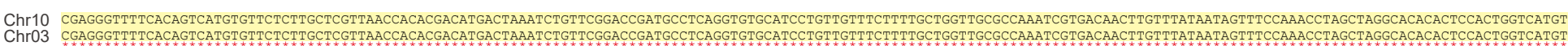

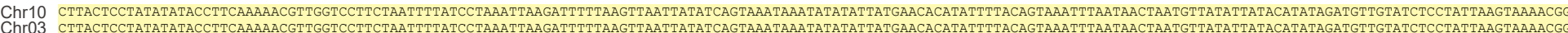

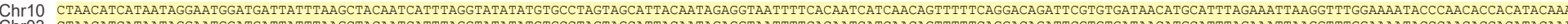

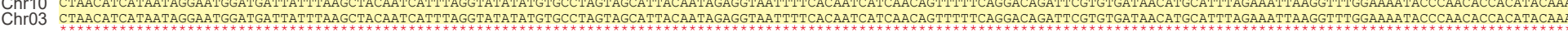

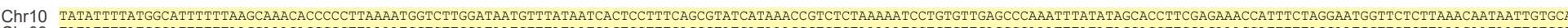

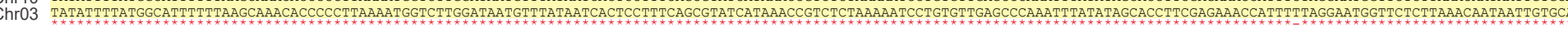

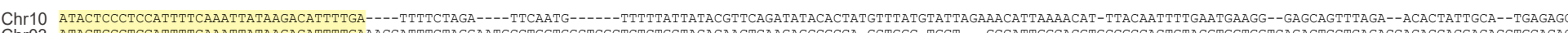

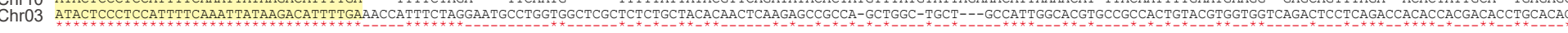

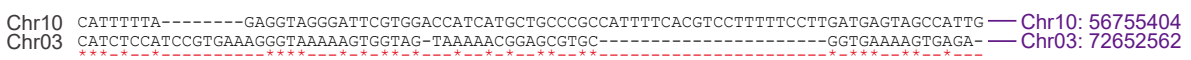

\section{Supplementary Figure 3}

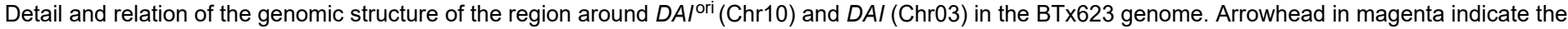
only base substitution (synonymous substitution) that exists between the CDS of $D A I^{\text {ori }}$ and that of DAI. 
bioRxiv preprint doi: https://doi.org/10.1101/2021.09.29.462495; this version posted October 2, 2021. The copyright holder for this preprint (which was not certified by peer review) is the author/funder. All rights reserved. No reuse allowed without permission.

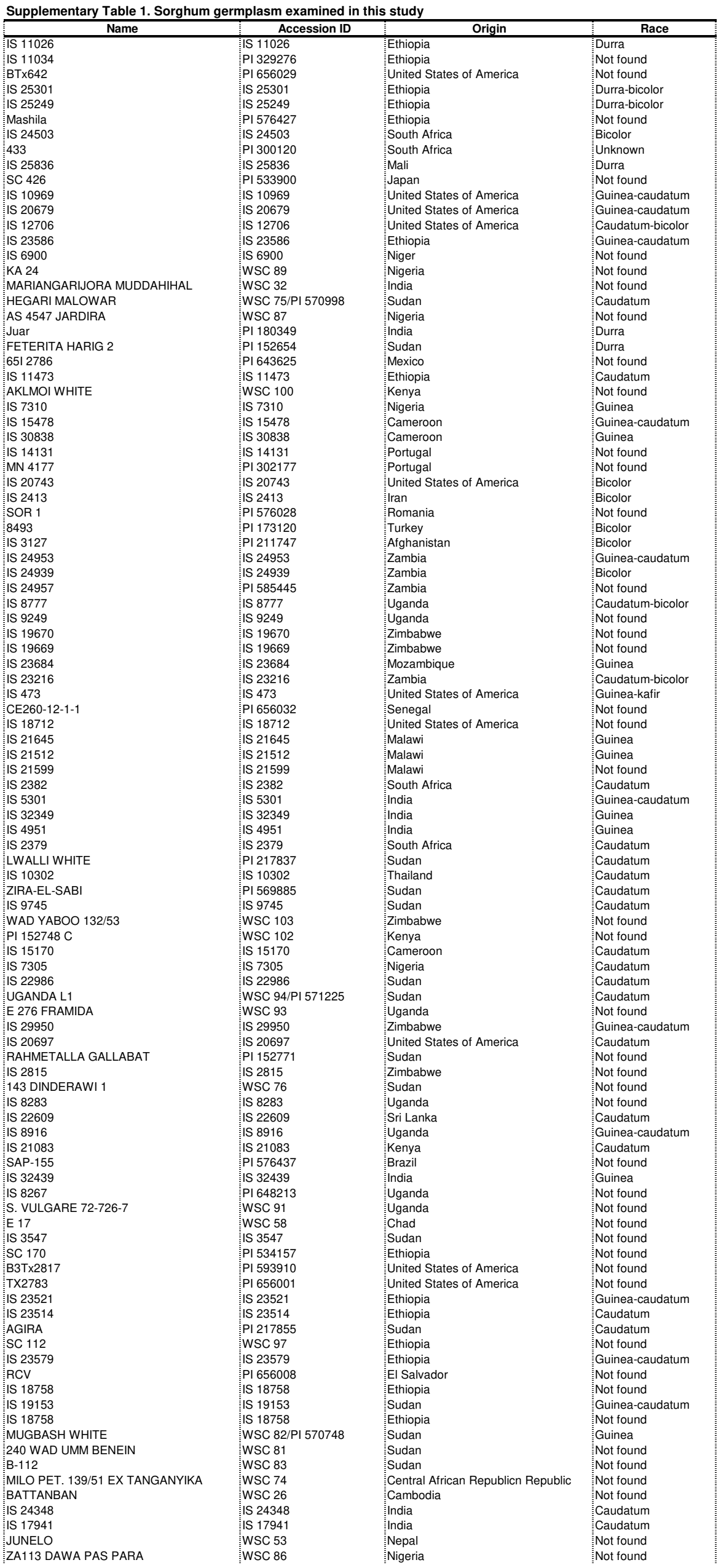


bioRxiv preprint doi: https://doi.org/10.1101/2021.09.29.462495; this version posted October 2, 2021. The copyright holder for this preprint (which was not certified by peer review) is the author/funder. All rights reserved. No reuse allowed without permission.

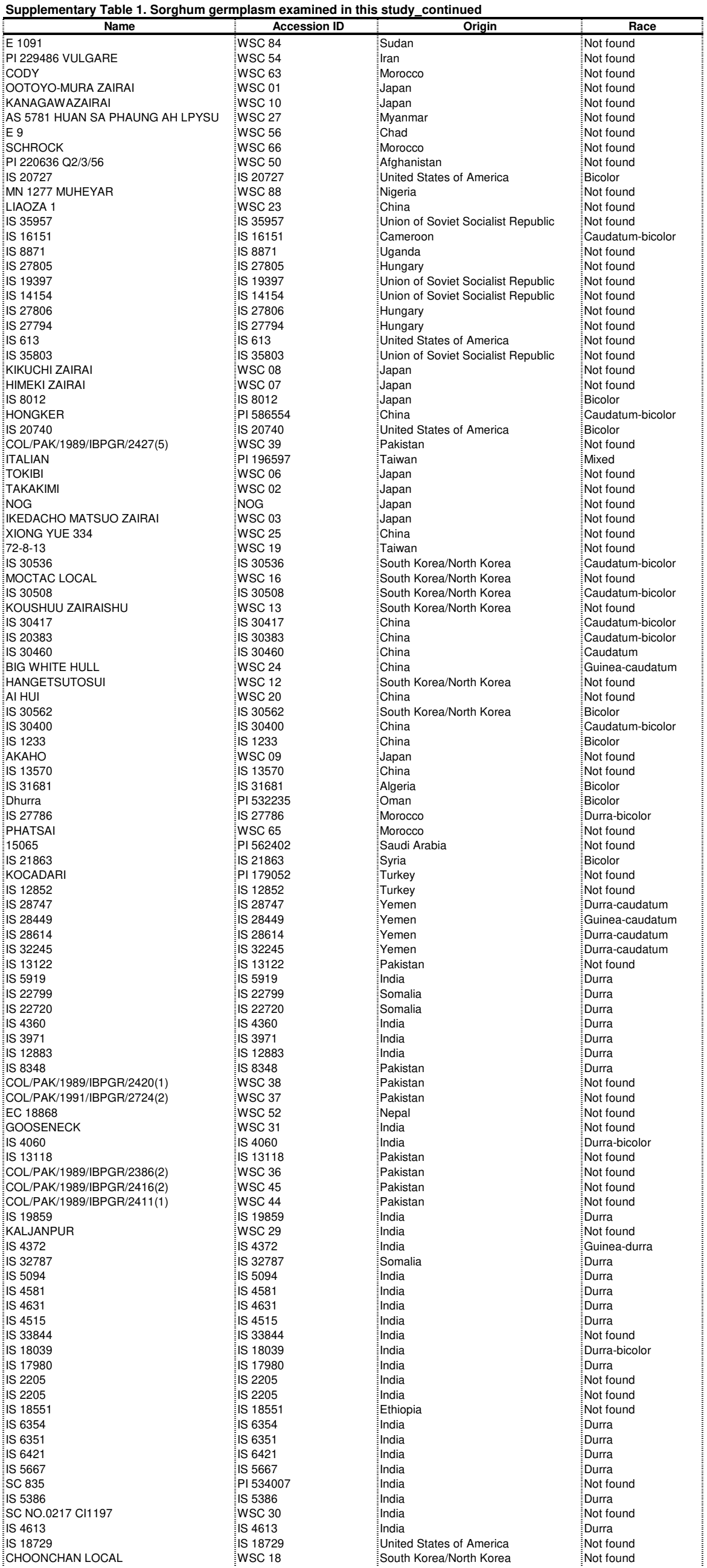


bioRxiv preprint doi: https://doi.org/10.1101/2021.09.29.462495; this version posted October 2, 2021. The copyright holder for this preprint (which was not certified by peer review) is the author/funder. All rights reserved. No reuse allowed without permission.

\begin{tabular}{|c|c|c|c|}
\hline Name & Accession ID & Origin & Race \\
\hline $\begin{array}{l}\text { SC } 56 \\
\text { IS } 20816\end{array}$ & $\begin{array}{l}\text { PI } 533910 \\
\text { IS } 20816\end{array}$ & Sudan & $\begin{array}{l}\text { Not found } \\
\text { Bicolor }\end{array}$ \\
\hline $\begin{array}{l}\text { IS } 20816 \\
\text { IS } 12447\end{array}$ & $\begin{array}{l}\text { IS } 20816 \\
\text { IS } 12447\end{array}$ & $\begin{array}{l}\text { United States of America } \\
\text { Sudan }\end{array}$ & $\begin{array}{l}\text { Bicolor } \\
\text { Durra-caudatum }\end{array}$ \\
\hline DHOOTI ANEHULA & WSC 33 & India & Not found \\
\hline B.T×2925 & PI 629053 & United States of America & Not found \\
\hline B.T $\times 2921$ & PI 629045 & United States of America & Not found \\
\hline BTx623 & PI 564163 & United States of America & Not found \\
\hline RTx432 & PI 656000 & United States of America & Not found \\
\hline B.T×2922 & PI 629047 & United States of America & Not found \\
\hline IS 10475 & IS 10475 & United States of America & Not found \\
\hline IS 24462 & IS 24462 & South Africa & Caudatum-bicolor \\
\hline AS 4637 NHORONGO NENPI & WSC 106 & United Republic of Tanzania & Not found \\
\hline IS 10288 & IS 10288 & United States of America & Not found \\
\hline AW 70/12 DL/59/1532 & WSC 69/PI 267512 & South Africa & Caudatum \\
\hline IS 19262 & IS 19262 & Sudan & Guinea-caudatum \\
\hline B2AR3043 & PI 576320 & United States of America & Not found \\
\hline CHAL WAXY SORGHUM & WSC 14 & South Korea/North Korea & Not found \\
\hline B3 BT×3197 & PI 598121 & United States of America & Not found \\
\hline PERSIAN DWARF & PI 576074 & United Kingdom & Not found \\
\hline E 959 & WSC 101 & Kenya & Not found \\
\hline IS 18757 & IS 18757 & Australia & Not found \\
\hline IS 3158 & IS 3158 & South Africa & Kafir \\
\hline IS 13893 & IS 13893 & South Africa & Kafir-caudatum \\
\hline IS 29335 & IS 29335 & Swaziland & Caudatum \\
\hline IS 29314 & IS 29314 & Swaziland & Durra-caudatum \\
\hline IS 27912 & IS 27912 & South Africa & Kafir-caudatum \\
\hline IS 29241 & IS 29241 & Swaziland & Kafir-caudatum \\
\hline IS 29187 & IS 29187 & Swaziland & Guinea-caudatum \\
\hline MORABA 74 & WSC 95/PI 563098 & Ethiopia & Kafir \\
\hline IS 13971 & IS 13971 & South Africa & Caudatum \\
\hline IS 24492 & IS 24492 & South Africa & Kafir \\
\hline IS 29606 & IS 29606 & South Africa & Kafir \\
\hline IS 22294 & IS 22294 & Botswana & Kafir \\
\hline IS 29733 & IS 29733 & Zimbabwe & Guinea-durra \\
\hline IS 20092 & IS 30092 & Zimbabwe & Durra-caudatum \\
\hline TSETA LOCAL NATURE TYPE $27 / 51$ & WSC 105 & Zimbabwe & Not found \\
\hline MN 401 & WSC 90 & Algeria & Not found \\
\hline KOURNIANIA & WSC 64 & Morocco & Not found \\
\hline IS 29304 & IS 29304 & Swaziland & Guinea-kafir \\
\hline IS 29233 & IS 29233 & Swaziland & Kafir \\
\hline IS 13872 & IS 13872 & South Africa & Not found \\
\hline IS 13870 & IS 13870 & South Africa & Not found \\
\hline IS 13869 & IS 13869 & South Africa & Not found \\
\hline IS 13868 & IS 13868 & South Africa & Not found \\
\hline IS 13782 & IS 13782 & South Africa & Kafir-durra \\
\hline IS 29269 & IS 29269 & Swaziland & Guinea-caudatum \\
\hline IS 8525 & IS 8525 & Ethiopia & Not found \\
\hline IS 29519 & IS 29519 & Lesotho & Kafir-caudatum \\
\hline IS 19445 & IS 19445 & Botswana & Kafir \\
\hline IS 3436 & IS 3436 & South Africa & Not found \\
\hline CAPRICORN & PI 586438 & Hungary & Kafir \\
\hline IS 12965 & IS 12965 & Cuba & Caudatum \\
\hline IS 12945 & IS 12945 & Nicaragua & Kafir \\
\hline EAR FROM PIETESBURG DL/60/107 & WSC 73 & South Africa & Not found \\
\hline THIMBA RED & PI 229877 & South Africa & Kafir-durra \\
\hline IS 19450 & IS 19450 & Botswana & Guinea-kafir \\
\hline IS 29441 & IS 29441 & Lesotho & Kafir-caudatum \\
\hline IS 29468 & IS 29468 & Lesotho & Guinea-caudatum \\
\hline IS 29358 & IS 29358 & Lesotho & Kafir \\
\hline NYAKASOBA BEST & WSC 61 & Lesotho & Not found \\
\hline IS 29565 & IS 29565 & Lesotho & Guinea-caudatum \\
\hline IS 29392 & IS 29392 & Lesotho & Kafir \\
\hline TENANT WHITE & WSC 60 & Lesotho & Kafir-caudatum \\
\hline IS 24463 & IS 24463 & South Africa & Kafir \\
\hline IS 29568 & IS 29568 & Lesotho & Kafir-caudatum \\
\hline IS 2389 & IS 2389 & South Africa & Kafir \\
\hline RED KAFIR & WSC 71 & South Africa & Not found \\
\hline E 233 BARNARD RED & WSC 70 & South Africa & Not found \\
\hline IS 2397 & IS 2397 & South Africa & Kafir \\
\hline IS 8774 & IS 8774 & South Africa & Kafir-durra \\
\hline S. BASUTORUM DL/60/97 & WSC 72 & South Africa & Not found \\
\hline IS 22616 & IS 22616 & Myanmar & Bicolor \\
\hline ALLAKH & WSC 51 & Bangladesh & Not found \\
\hline COL/PAK/1989/BPGR/2550(1) & WSC 42 & Pakistan & Not found \\
\hline ITALIAN & PI 295123 & Australia & Kafir \\
\hline COL/PAK/1989/BPGR/2553(4) & WSC 43 & Pakistan & Not found \\
\hline IS 10646 & IS 10646 & United States of America & Not found \\
\hline ESHOME & WSC 67 & South Africa & Not found \\
\hline IS 29627 & IS 29627 & South Africa & Durra-caudatum \\
\hline IS 22239 & IS 22239 & Botswana & Kafir \\
\hline IS 14290 & IS 14290 & Botswana & Kafir-durra \\
\hline IS 29326 & IS 29326 & Swaziland & Caudatum-bicolor \\
\hline IS 26701 & IS 26701 & South Africa & Caudatum-bicolor \\
\hline RIO & PI 651496 & - & Not found \\
\hline IS 27887 & IS 27887 & South Africa & Caudatum-bicolor \\
\hline IS 24453 & IS 24453 & South Africa & Caudatum-bicolor \\
\hline
\end{tabular}


bioRxiv preprint doi: https://doi.org/10.1101/2021.09.29.462495; this version posted October 2, 2021. The copyright holder for this preprint (which was not certified by peer review) is the author/funder. All rights reserved. No reuse allowed without permission.

Supplementary Table 2. Primers used in this study

\begin{tabular}{|c|c|c|c|}
\hline No. & Primer name & Sequence $\left(5^{\prime}->3^{\prime}\right)$ & Comment \\
\hline 1 & gDAl_genotyping_fw & GACCACATGGGCCTTCTTTA & \multirow{2}{*}{$\begin{array}{l}\text { For genotyping of DAl gene using BTx623, } \\
\text { NOG, and selected germplasm genomic DNA }\end{array}$} \\
\hline 2 & gDAl_genotyping_rv & TGGCCCTTTGATAAGTCGAG & \\
\hline 3 & TOPO_DAl_fw & caccATGCAGGTCGGAGGAGGAGC' & \multirow{6}{*}{$\begin{array}{l}\text { For constructing "Free GFP" and "DAI-GFP" } \\
\text { plasmids }\end{array}$} \\
\hline 4 & DAl-tag_rv & GTTGAACACCGACAGCGGCA & \\
\hline 5 & Just_linker_fw & caccAAGGGTGGG & \\
\hline 6 & Just_linker_rv & CССАСССТT & \\
\hline 7 & M13 fw & GTAAAACGACGGCCAG & \\
\hline 8 & M13 rv & CAGGAAACAGCTATGAC & \\
\hline 9 & Linearize_pBUH3_fw & TTGGGTACCAGCTCGAATTT & \multirow{10}{*}{$\begin{array}{l}\text { For constructing "DAlpro::DAl" plasmid for } \\
\text { making sorghum transgenic plants }\end{array}$} \\
\hline 10 & Linearize_pBUH3_rv & CTCTAGAGAGGGGCACGAC & \\
\hline 11 & pBUH3_DAlpro_inF_fw & TGCCCCTCTCTAGAGGAATGAACAAGGACCGCAT & \\
\hline 12 & pBUH3_DAI_inF_rv & CGAGCTGGTACCCAACCTACCTCGCCTGGCTAT & \\
\hline 13 & $\mathrm{pBUH} 3$ inseq $\mathrm{fw}$ & TCGTATGTTGTGTGGAATTG & \\
\hline 14 & pBUH3_inseq_rv & AATGTATAATTGCGGGACTC & \\
\hline 15 & DAI_C_fw & CAGTTCCTGCACGGGTTCAG & \\
\hline 16 & LINE_rv & TCCATATGTCACACCAGGGG & \\
\hline 17 & DAl_1st_fw & GATTCGTCTCGTCAATTCCG & \\
\hline 18 & DAI_2nd_fw & CCTCAGAGGAGCCGTACTTG & \\
\hline 19 & DAl_icheck_fw & GCACCAGCATCACTTCTTCA & \multirow[b]{2}{*}{ For genotyping of the transgenic sorghum } \\
\hline 20 & DAI_pBUH3_icheck_rv & TTGCGGGACTCTAATCATAAAAA & \\
\hline 21 & Cont_H2A_qfw & GCCAGGGACAACAAGAAGAC & \multirow{4}{*}{$\begin{array}{l}\text { For qPCR to determine the copy number of the } \\
\text { transgene }\end{array}$} \\
\hline 22 & Cont_H2A_qrv & GACACCGGACAGCAGCTT & \\
\hline 23 & fDAl gfw & АTTTCGGCTCCAACAATGTC & \\
\hline 24 & fDAI_qrv & GATGTTGGCGACCTCGTATT & \\
\hline 25 & DAlpro_1st_fw & TGATGGTATGCGGTGTTTGT & \multirow{9}{*}{$\begin{array}{l}\text { For constructing "SbDAl" and "gNOG" } \\
\text { plasmids for making Kasalath transgenic plants }\end{array}$} \\
\hline 26 & DAI3UTR_1st_rv & GTAGATGGCACGGGCTGTAT & \\
\hline 27 & DAlpro_nest_inF_fw & ATGTATGATAATTCGGAATGAACAAGGACCGCATT & \\
\hline 28 & DAltag_nest_inFrv & СTCCCGGCCGCCATGCTACCTCGCCTGGCTATACG & \\
\hline 29 & gNOG_inF_rv & CTCCCGGCCGCCATGAAATCCACCGACGACAGAAG & \\
\hline 30 & icheck_fw2 & TGCAATAGGTCAGGCTCTCG & \\
\hline 31 & icheck_rv & TATCTGGGAACTACTCACAC & \\
\hline 32 & DAlseq_fw & TTСТTСТTСТTСССАСТTTC & \\
\hline 33 & DAlseq_fw2 & CTTCGTCGTCCCAGTCGT & \\
\hline 34 & gNOG_icheck_fw & TGTTTCCCTGATGACACCTC & \multirow{2}{*}{ For genotyping of the transgenic Kasalath } \\
\hline 35 & DAl_icheck_rv & GCAGGTCACTGGATTTTGGT & \\
\hline 36 & 1stM_01_fw & CGCGATAAGGAAATTTGGAG & \multirow{34}{*}{$\begin{array}{l}\text { For genotyping to establish NILs for } \\
9 A L 3 / q A P 3\end{array}$} \\
\hline 37 & 1stM_01_rv & GAACCTTGCCTTGCCTTACA & \\
\hline 38 & 1stM_02_fw & СTCACGCCACGAAAATATCA & \\
\hline 39 & 1stM_02_rv & GAGGGAGATGGAGGTGGTG & \\
\hline 40 & 1stM_03_fw & AGGAAAGGGAGGTGAGGTGT & \\
\hline 41 & 1stM_03_rv & AATAAAAGGCGCACACCAAG & \\
\hline 42 & 1stM_04_fw & TATACCACCCACACCCAGCA & \\
\hline 43 & 1stM_04_rv & СТАССССТСАССАСАGATCC & \\
\hline 44 & 1stM_05_fw & GGATTGCGGTAAGATCATCA & \\
\hline 45 & 1stM_05_rv & CCGTGATTGGACAATGTTTG & \\
\hline 46 & 1stM_06_fw & AACCAGACCACTGCTGGTG & \\
\hline 47 & 1stM_06_rv & ATTCTGTCGGAGGCATCATC & \\
\hline 48 & 1stM_07_fw & GGCTCCAATCAACTTGGACT & \\
\hline 49 & 1stM_07_rv & CGGCACCATGCAGTAAATAA & \\
\hline 50 & 1stM_08_fw & GGAGAGTCAATGTATCTCAGGTTTG & \\
\hline 51 & 1stM_08_rv & TTGGATGGGCTAATTCTCCA & \\
\hline 52 & 1stM_09_fw & ACCGACTCGTCGCATACTTC & \\
\hline 53 & 1stM_09_rv & CACAGTGCACACACCGTTT & \\
\hline 54 & 1stM_10_fw & ТстСССтTСтССССтстTст & \\
\hline 55 & 1stM_10_rv & CGGAAAGGCTACCTCTCTCTC & \\
\hline 56 & 1stM_11_fw & GCCGCTATTACCCTAGCAGA & \\
\hline 57 & 1stM_11_rv & AGCAACAGCAAAGCGTAGGT & \\
\hline 58 & 1stM_07.5_fw & TTTAGGGACGGAGGGAGTAA & \\
\hline 59 & 1stM_07.5_rv & CTGATCCATCATCGCTTTCA & \\
\hline 60 & $1 \mathrm{stM} \_08.2 \_\mathrm{fw}$ & ACACGGTCATTAGGCGACAT & \\
\hline 61 & 1stM_08.2_rv & TGCGACCTTTTGGCTAGACT & \\
\hline 62 & 1stM_08.5_fw & AGCCATGAGCAAGCAAAAA & \\
\hline 63 & $1 \mathrm{stM}$ 08.5_rv & TCTGCGCACTCTTTCATCAC & \\
\hline 64 & 1stM_09.5_fw & AGGAACAGATGCGAGAGGAA & \\
\hline 65 & $1 \mathrm{stM}$ 09.5_rv & TTGTTGTGAACGATCCGACT & \\
\hline 66 & 1stM_12_fw & CCGGTAAGATCCCATTAGGG & \\
\hline 67 & 1stM_12_rv & CCAACCGTGGATTTAGGAGA & \\
\hline 68 & 1stM_13_fw & GCCTCGAGCAGACCTACG & \\
\hline 69 & 1stM_13_rv & TACGAGGACGAGGAGGGAGT & \\
\hline
\end{tabular}

